\author{
AUTHOR'S POST PRINT (Romeo Colour: Green) \\ Journal of Computational Physics (ISSN: 0021-9991), 180 (2): 751-774 (2002). \\ DOI:10.1006/jcph.2002.7131 \\ Publisher version available at \\ http://www.sciencedirect.com/science/article/pii/S0021999102971317
}

\title{
3D analysis of crystal/melt interface shape and Marangoni flow instability in solidifying liquid bridges
}

\author{
M. Lappa* and R. Savino \\ Università degli Studi di Napoli "Federico II" \\ Dipartimento di Scienza e Ingegneria dello Spazio "Luigi G. Napolitano" \\ P.le V.Tecchio 80, 80125 Napoli (Italy) \\ *current e-mail address: marcello.lappa@strath.ac.uk
}

running title: flow instability in solidifying liquid bridges

\begin{abstract}
Solidification of Gallium $(\mathrm{Pr}=0.02)$ in liquid bridges in zero gravity conditions is investigated by numerical solutions of the three-dimensional and time-dependent flow-field equations. A single region (continuum) formulation based on the enthalpy method is adopted to model the phase change problem. The paper analyzes the influence of the azimuthally asymmetric and steady first bifurcation of the Marangoni flow on the shape of the solid/melt interface during the crystal growth process. The numerical results show that this interface is distorted in the azimuthal direction. The distortion is related to the sinusoidal three-dimensional temperature disturbances due to the instability of the Marangoni flow. The three-dimensional flow field organization, related to the wave number, changes during the solidification process; this behaviour is explained according to the variation of the aspect ratio of the solidifying liquid bridge. A correlation law is found for the azimuthal wave number of the instability as function of the melt zone aspect ratio.
\end{abstract}

Key words: liquid bridge, flow instability, crystal growth

Categories: 65M06, 76D05, 76E15 


\section{Introduction}

Single crystals of elemental and compound semiconductors play an important role as basic materials for electronic and optoelectronic device applications. The float zone method is an important technique to produce high quality crystal material. By the containerless method, crystals can be grown with less contamination, more homogeneity and higher purity. In these techniques the melt is positioned and solidified without physically contacting the container's wall, in order to minimize container-induced contamination and heterogeneous nucleation.

In the float zone crystal growth, a melt zone is produced by heating a short length of feed rod by a heat source such as a ring heater. The rod is slowly moved through the hot zone, and the crystal is obtained by resolidification of the melt as it is moved away from the melt zone. If the melt/crystal interface remains flat during the resolidification, often uniform and high quality single crystals can be produced. In particular the quality of crystals is highly dependent upon the uniformity of the thermal field at the solidifying interface and hence the on the convection fields there [1].

Microgravity gives the possibility to avoid some limitations related to the ground environment, e.g. buoyancy driven convection, that is one of the most important causes of imperfections and crystal defects. Moreover in zero-g conditions it is possible to form very large floating zones. During recent years, the availability of Sounding Rockets and orbiting laboratories as the Spacelab, has made possible microgravity experiments and manufacturing processing, which could not be performed on Earth under normal gravity conditions. However, even in microgravity the presence of free-melt-gas interface at different temperatures and hence of surface tension gradients induces Marangoni convection. Typically liquid metals are opaque and at large temperature, so that it is hard to visualize and to study Marangoni flow in real floating zones. Other difficulties in the analysis of Marangoni flow in real floating zones are that, due to phase change related to melting and solidification of the material, the geometry of the liquid volume is not known a priori. This led the investigators to introduce in the middle 70 s the so-called "half zone" model for the study of the features of the Marangoni flow in floating zones. The flow situation in this model corresponds (approximately) to that of half of a floating zone, heated radially by a ring heater positioned on the equatorial plane around the zone. 
Experiments [2] performed with transparent liquids (with Prandtl numbers higher than those typical of liquid metals) have shown that for sufficiently small values of the Marangoni number, the convection in the liquid column is laminar, steady and axisymmetrical, but when the Marangoni number exceeds certain critical values depending on the Prandtl number of the liquid, on the geometry and on the boundary conditions, the liquid motion can undergo a transition to an oscillatory three-dimensional complex flow pattern. The appearance of these instabilities provides a possible explanation to justify the presence of undesirable macroscopic and microscopic imperfections in the final crystals obtained in microgravity conditions.

A number of theoretical and numerical studies have been carried out based on the linear stability theory $[3,4]$. The development of supercomputers and efficient numerical methods led the investigators to study the problem through direct numerical solution of the non linear and timedependent Navier Stokes equations. Rupp et al. [5] and Levenstan and Amberg [6], Imaishi et al. [7], Yasushiro et al. [8], Leypoldt et al. [9] found that for liquid metals the first bifurcation is stationary (i.e. the supercritical three-dimensional state is steady) and that the regime becomes oscillatory only when the Marangoni number is further increased (second oscillatory bifurcation). Lappa and Savino [10] and Lappa et al. [11] used parallel supercalculus to study the azimuthal structure (characterized by the appropriate value of the azimuthal wave number of the instability) of the flow pattern that is established after the first Marangoni flow bifurcation. They analyzed the influence of the aspect ratio and of the non-cylindrical (convex or concave) interface shape. Shevtsova et al. [12], Lappa et al. [13] and Lappa et al. [14] analyzed the features of the Marangoni flow instability for high Prandtl number liquids. Comparison of the linear stability results with the computational results have confirmed that for high Prandtl numbers the instability is oscillatory (Hopf bifurcation) whereas for low Prandtl numbers the instability breaks the spatial axisymmetry (but the flow regime is still steady) prior to the onset of time dependent flow field (in this case the instability is hydrodynamic in nature i.e. its mechanism does not involve a coupling between the temperature and the velocity disturbances).

Since it is very difficult to do well controlled experiments with liquid metals of small Prandtl numbers (due to opacity, reactivity and high temperatures of the melts), there are only few experiments on the flow instability in half zone liquid bridges of semiconductor materials [15-20]. 
Nakamura et al. [17] and Hibiya et al. [19] studied the Marangoni flow azimuthal organization in a half zone of Silicon $(\operatorname{Pr}=0.02$, diameter $\mathrm{D}=1[\mathrm{~cm}])$ under highly supercritical conditions using a $\mathrm{X}$ ray radiography with zirconium-core tracers. During a sounding rocket mission and other parabolic flights they observed that when the column height was short (column formation period), the tracer particles preferred to stay at positions 90 degree apart from each other, while after a Silicon column with full height was formed, tracer particles preferred to stay at positions of 180 degree apart from each other. This behaviour was interpreted as a change of the azimuthal wave number during the melting process. To better analyze the above phenomena an "ideal" solidification process of pure Gallium in half zone liquid bridges in zero g conditions, is numerically simulated in the present paper. Gallium is chosen for the simulation due to the low melting point that makes it suitable for future experimental investigations at relatively small temperatures (close to the ambient temperature).

\section{Physical and mathematical model}

\section{$\underline{2.1 \text { Basic assumptions }}$}

The geometry of the problem is shown in Fig. 1a. At the instant $\mathrm{t}=0$, a cylindrical liquid bridge of length $\mathrm{L}$ and diameter $\mathrm{D}$ is held between two coaxial disks at different temperatures. The upper disk in Fig.1a is kept at the temperature $\mathrm{T}_{\mathrm{H}}$ higher than the temperature $\mathrm{T}_{\mathrm{C}}$ of the lower cold disk. The solidification takes place as the temperature $T_{C}$ is decreased to a value which is less than the melting temperature $T_{m}$. The instantaneous distance from the disk at temperature $T_{H}$ of the point given by the intersection of the crystal-melt interface and of the liquid bridge symmetry axis is denoted as $\mathrm{L}_{\mathrm{i}}$ (Fig. 1b). The ratio $\mathrm{A}_{\mathrm{i}}=\mathrm{L}_{\mathrm{i}} / \mathrm{D}$ is denoted as the "melt aspect ratio". The temperature difference imposed at $\mathrm{t}=0$ is denoted by \#\# .

The liquid phase is assumed homogeneous and Newtonian, with constant density and transport coefficients. The liquid-gas interface is characterized by a surface tension $\sigma$ exhibiting a linear decreasing dependence on the temperature:

$$
\sigma=\sigma_{\mathrm{o}}-\sigma_{\mathrm{T}}(\mathrm{T}-\mathrm{To})
$$


where $\sigma_{\mathrm{O}}$ is the surface tension for $\mathrm{T}=\mathrm{To} ; \sigma_{\mathrm{T}}$ is the negative rate of change of the surface tension with temperature $\left(\sigma_{\mathrm{T}}=-\mathrm{d} \sigma / \mathrm{dT}>0\right)$.

\subsection{Phase change modeling theory}

Due to the absorption or release of latent energy, phase change problems are nonlinear. The numerical procedures able to solve these problems can be divided into two groups:

1) Multiple region solutions, that utilize independent equations for each phase and couple them with appropriate boundary conditions at the solid/liquid interface. Difficulties arise when this technique is employed since the governing equations are based on the classical Stefan formulation, i.e., a temperature-based heat transfer equation. In this case in the vicinity of the phase change, conditions on temperature, velocity, and latent heat evolution have to be accounted for. This effectively rules out the application of a fixed-grid numerical solution, as deforming grids or transformed coordinate systems are required to account for the position of the phase front.

2) Single region (continuum) formulations which eliminate the need for separate equations in each phase, by establishing conservation equations which are universally valid. The major advantage of the single region formulations is that they do not require to consider interface motion and boundary conditions internal to the solidifying domain and hence, the associated use of quasi-steady approximations, numerical remeshing and coordinate mapping. In the present paper the continuum model (single region formulation) is adopted (see Ref. [21-24]).

Assumptions invoked in the development of equations for this continuum model include: laminar flow, Newtonian behaviour of the phases (this implies that solids, should be treated as highly viscous fluids), constant phase densities, local thermodynamic equilibrium. In addition the density $\rho$ and the specific heat $\mathrm{Cp}$ are assumed to be equal in the solid and the liquid phases.

Moreover the solid phase is assumed to be nondeforming and free of internal stress, while the mushy multiphase region (region where phase change occurs) is viewed as a porous solid characterized by an isotropic permeability $\eta$ (see Voller et al. [21], Bennon and Incropera [22] and Brent et al. [24]). 
The enthalpy method is used to solve the temperature field over the computational domain including both the solid and liquid phases.

\subsection{The enthalpy method}

The so-called "enthalpy formulation", which allows a fixed-grid solution to be undertaken, removes the need to explicitly satisfy conditions at the phase front and is therefore able to utilize standard solution procedures for the fluid flow and energy equations directly, without resorting to mathematical manipulations and transformations. The enthalpy methods account for the latent heat in the energy equations by assigning a nodal latent heat value to each computational cell according to the temperature of the cell. Upon changing phase, the nodal latent heat content of the cell is adjusted to account for latent heat absorption or evolution, this adjustment being reflected in the energy equation as either a source or sink (a distinctive advantage of this arrangement is that no explicit conditions for energy conservation at the solid/liquid interface need to be accounted for). Latent heat during phase change is incorporated in the energy equation using for each phase the following definition of enthalpy:

$\mathrm{h}=\mathrm{CpT}+\Delta \mathrm{H}$

where $\Delta \mathrm{H}=\beta \mathrm{L}_{\mathrm{m}}, \mathrm{L}_{\mathrm{m}}$ is the latent heat, $\mathrm{Cp}$ the specific heat coefficient and $\beta$ the liquid fraction defined as

$\beta=0$ for $\mathrm{T}<\mathrm{T}_{\text {sol }}$

$\beta=\frac{T-T_{\text {sol }}}{T_{\text {liq }}-T_{\text {sol }}}$ for $T_{\text {sol }} \leq \mathrm{T} \leq \mathrm{T}_{\text {liq }}$

$\beta=1$ for $\mathrm{T}>\mathrm{T}_{\text {liq }}$

$\mathrm{T}_{\mathrm{sol}}=\mathrm{T}_{\mathrm{m}}-\Delta \mathrm{T}_{\mathrm{m}}$ and $\mathrm{T}_{\text {liq }}=\mathrm{T}_{\mathrm{m}}+\Delta \mathrm{T}_{\mathrm{m}}, 2 \Delta \mathrm{T}_{\mathrm{m}}$ is a temperature interval chosen to represent the range over which phase change occurs $\left(\mathrm{T}_{\text {liq }}\right.$ is the liquidus temperature at which solid formation commences and $\mathrm{T}_{\text {sol }}$ is the temperature at which full solidification is achieved, see Voller et al. [21]). An extended temperature range is considered for solidification. From a "physical" point of view this assumption is based on the idea (Voller et al. [21] and Bennon and Incropera [22]), that in 
many cases solid formation occurs as a permeable crystalline-like matrix which coexists with the liquid phase (mushy region). From a numerical point of view a linear approximation is considered for the liquid fraction since a step change in the liquid fraction could cause serious numerical instabilities. Further details are reported in Section 3.2.

During solidification, latent energy is released at the interfaces which separate the phases within the mushy region. Thus the energy equation can be written as:

$$
\frac{\partial \rho h}{\partial t}+\frac{\partial \rho\left(\beta L_{m}\right)}{\partial t}=-\underline{\nabla} \cdot[\rho \underline{V h}]+k \nabla^{2} T
$$

where $\mathrm{k}$ is the thermal conductivity; the unsteady latent heat content term on the left side of (2b) is equal to zero for $\mathrm{T}<\mathrm{T}_{\text {sol }}$ and $\mathrm{T}>\mathrm{T}_{\text {liq }}$ (it influences the energy equation only within the mushy region).

Equation (2b), together with the continuity and Navier-Stokes equations, can be posed in nondimensional form scaling the lengths by the axial distance between the circular disks (L) and the velocity by the energy diffusion velocity $\mathrm{V}_{\alpha}=\alpha / \mathrm{L}$ ( $\alpha=\mathrm{K} / \rho \mathrm{Cp}$ is the thermal diffusivity); the scales for time and pressure are, respectively, $\mathrm{L}^{2} / \alpha$ and $\rho \alpha^{2} / \mathrm{L}^{2}$.

The non-dimensional conservative form reads :

$\underline{\nabla} \cdot \underline{\mathrm{V}}=0$

$\frac{\partial \underline{\mathrm{V}}}{\partial \mathrm{t}}=-\underline{\nabla \mathrm{p}}-\underline{\nabla} \cdot[\underline{\mathrm{V}} \underline{\mathrm{V}}]+\operatorname{Pr} \nabla^{2} \underline{\mathrm{V}}-\operatorname{Pr} \frac{1}{\eta} \underline{\mathrm{V}}$

$\frac{\partial \mathrm{T}}{\partial \mathrm{t}}+\frac{\mathrm{L}_{\mathrm{m}}}{\mathrm{Cp}} \frac{\partial \beta}{\partial \mathrm{t}}=-\underline{\nabla} \cdot[\underline{\mathrm{VT}}]+\nabla^{2} \mathrm{~T}$

where $\mathrm{V}$ and $\mathrm{p}$ are the non-dimensional velocity and pressure, $\mathrm{Pr}$ is the Prandtl number, defined by $\operatorname{Pr}=v / \alpha(v=\mu / \rho$ is the kinematic viscosiy $)$.

An important problem with fixed-grid solution procedures, is accommodating the zero velocity condition, which is required as a liquid region turns to solid. Various methods (see Brent et al. [24]) have been used to "switch off" velocities in computational cells that are freezing (or "switch on" velocities in the case of melting). Velocities may simply be set to zero. Another possible approach is based on the viscosity. The viscosity of a cell is driven to a very large value as the latent heat content of the cell falls to zero. The increasing viscosity provides the necessary coupling between 
the physical state of the material in the cell and the momentum equations, thereby driving velocities to zero in cells that are solid. A third possible approach (enthalpy-porosity approach) requires that computational cells that are undergoing a phase change are modeled as a pseudo-porous media, with the porosity $\eta$ being a function of $\Delta H$ ranging between 1 (fully liquid cell) and 0 (fully solid cell). This approach has been used for the present computations. The term $-\operatorname{Pr} \frac{1}{\eta} \underline{V}$ in equation (3b) is the Darcy term (see Bennon and Incropera [23] and Brent et al. [24]) added to the momentum equation to eliminate convection in the solid phase. In the present analysis permeability is assumed to vary according to the Carman-Kozeny equation (Bennon and Incropera [23] and Brent et al. [24])

$$
\eta=\frac{\beta^{3}}{(1-\beta)^{2}}
$$

In the pure solid $(\beta=0)$ and pure liquid $(\beta=1)$, equation $(3 b)$ reduces to the appropriate limits, namely $\eta=0$ and $\eta=\infty$ respectively. In practice the effect of $\eta$ is as follows: in full liquid elements $1 / \eta$ is zero and has no influence; in elements that are changing phase, the value of $1 / \eta$ will dominate over the transient, convective and diffusive components of the momentum equation, thereby forcing them to imitate Carman-Kozeny law; in totally solid elements, the final large value of $1 / \eta$ will swamp out all terms in the governing equations and force any velocity predictions effectively to zero.

Since each of the continuum equations is valid throughout the entire domain, explicit consideration need not be given to boundaries between solid, multiphase and liquid regions.

\subsection{Initial and boundary conditions}

The initial conditions are:

$\mathrm{t}=0: \quad \underline{\mathrm{V}}(\mathrm{z}, \mathrm{r}, \varphi)=0, \mathrm{~T}(\mathrm{z}, \mathrm{r}, \varphi)=\mathrm{T}_{\text {liq }}+\mathrm{z} \Delta \mathrm{T}$

i.e. the liquid is motionless and the temperature is $\mathrm{T}_{\mathrm{C}}=\mathrm{T}_{\text {liq }}$ on the cold disk and $\mathrm{T}_{\mathrm{H}}=\mathrm{T}_{\text {liq. }}+\Delta \mathrm{T}$ on the hot disk.

For $\mathrm{t}>0$, no slip conditions are considered for both the supporting disks. When, for $\mathrm{t}>\mathrm{t}^{*}$ the solidification process starts ( $\mathrm{t}^{*}$ is the instant at which solidification takes place), the thermal boundary conditions on the disks are 
$\mathrm{T}_{\mathrm{C}}=\mathrm{T}_{\text {liq }}-\mathrm{c}\left(\mathrm{t}-\mathrm{t}^{*}\right)$

where $\mathrm{c}$ is the ramping rate

on the hot disk

$0 \leq \mathrm{r} \leq \mathrm{R} / \mathrm{L} ; 0 \leq \varphi \leq 2 \pi$

$\mathrm{T}_{\mathrm{H}}=\mathrm{T}_{\text {liq }}+\Delta \mathrm{T}$

the kinematic condition of stream surface (zero normal velocity), the Marangoni conditions (shear stress balance) and the adiabatic condition are applied on the cylindrical interface:

on the cylindrical free surface $\quad 0 \leq \mathrm{z} \leq 1 ; 0 \leq \varphi \leq 2 \pi$

$\mathrm{V}_{\mathrm{r}}(\mathrm{z}, \mathrm{r}=\mathrm{R} / \mathrm{L}, \varphi, \mathrm{t})=0$

$\frac{\partial V_{\mathrm{Z}}}{\partial \mathrm{r}}(\mathrm{z}, \mathrm{r}=\mathrm{R} / \mathrm{L}, \varphi, \mathrm{t})=-\mathrm{Ma}_{\mathrm{r}} \frac{\partial \mathrm{T}}{\partial \mathrm{z}}(\mathrm{z}, \mathrm{r}=\mathrm{R} / \mathrm{L}, \varphi, \mathrm{t}) \beta(\mathrm{z}, \mathrm{r}=\mathrm{R} / \mathrm{L}, \varphi, \mathrm{t})$

$\mathrm{r} \frac{\partial \mathrm{V}_{\varphi}}{\partial \mathrm{r}}(\mathrm{z}, \mathrm{r}=\mathrm{R} / \mathrm{L}, \varphi, \mathrm{t})-\mathrm{V}_{\varphi}(\mathrm{z}, \mathrm{r}=\mathrm{R} / \mathrm{L}, \varphi, \mathrm{t})=-\mathrm{Ma}_{\mathrm{r}} \beta(\mathrm{z}, \mathrm{r}=\mathrm{R} / \mathrm{L}, \varphi, \mathrm{t}) \frac{\partial \mathrm{T}}{\partial \varphi}(\mathrm{z}, \mathrm{r}=\mathrm{R} / \mathrm{L}, \varphi, \mathrm{t})$

$\frac{\partial \mathrm{T}}{\partial \mathrm{r}}(\mathrm{z}, \mathrm{r}=\mathrm{R} / \mathrm{L}, \varphi, \mathrm{t})=0$

where the reference Marangoni number $\mathrm{Ma}_{\mathrm{r}}$ is defined as $\mathrm{Ma}_{\mathrm{r}}=\sigma_{\mathrm{T}} \mathrm{L} / \mu \alpha$.

The Marangoni number corresponding to the applied temperature difference is computed as $\mathrm{Ma}=\Delta \mathrm{T} \cdot \mathrm{Ma}_{\mathrm{r}}=\Delta \mathrm{T} \sigma_{\mathrm{T}} \mathrm{L} / \mu \alpha$; further to the Marangoni number, the instantaneous Marangoni number is defined as $M a_{\mathrm{i}}=\Delta \mathrm{T} \sigma_{\mathrm{T}} \mathrm{L}_{\mathrm{i}} / \mu \alpha$.

\section{Numerical solution}

The equations (3a-c) and the initial and boundary conditions (6-7) were solved numerically in cylindrical co-ordinates in primitive variables by a control volume method. The domain was discretized with a non uniform but structured axisymmetric mesh and the flow field variables defined over a staggered grid. The conservation laws have been written for an arbitrary spatial 
domain $\Omega$ bounded by a surface $\partial \Omega$. Integrating over the generic control volume and using the Gauss theorem to transform volume integrals in surface integrals, the equations read

$$
\begin{aligned}
& \underline{V}^{n+1}=\underline{V}^{n}+\Delta t \frac{1}{\Omega}\left[-\int_{\Omega \Omega}[\underline{V} \underline{V}] \cdot \underline{n} d S+\operatorname{Pr} \int_{\alpha \Omega}[\underline{\nabla} \underline{V}] \cdot \underline{n} d S\right]^{n}-\Delta t \frac{1}{\Omega} \int_{\Omega} \underline{\nabla} p^{n} d \Omega-\Delta t \frac{1}{\Omega} \operatorname{Pr} \frac{1}{\eta} \int_{\Omega} \underline{V}^{n+1} d \Omega \\
& T^{n+1}=T^{n}+\Delta t \frac{1}{\Omega}\left[-\int_{\alpha \Omega}[\underline{V} T] \cdot \underline{n} d S+\int_{\Omega}[\underline{\nabla} T] \cdot \underline{n} d S\right]^{n} \frac{1}{\xi}
\end{aligned}
$$

where

$\xi=1+\frac{\mathrm{L}_{\mathrm{m}}}{\mathrm{Cp}} \frac{1}{\mathrm{~T}_{\text {liq }}-\mathrm{T}_{\text {sol }}}$ for $\mathrm{T}_{\text {sol }} \leq \mathrm{T} \leq \mathrm{T}_{\text {liq }}$

$\xi=1$ for $\mathrm{T}<\mathrm{T}_{\text {sol }}$ and $\mathrm{T}>\mathrm{T}_{\text {liq }}$

The problem is solved with the well known Marker and Cell method (see e.g. Lappa and Savino [10] and Fletcher [25]).

\section{$\underline{\text { 3.1 Marangoni flow and grid stretching technique }}$}

The computations have been performed using non-uniform grids. A finer grid has been introduced near the free surface to locally enhance the resolution. The number of points clustered near the free surface are specified using the notation $\mathrm{Nz} \times\left(\mathrm{Nr}_{\mathrm{b}}+\mathrm{Nr}_{\mathrm{S}}\right)$ (where $\mathrm{Nr}_{\mathrm{s}}$ is the number of points stretched near the free surface and $\mathrm{Nr}_{\mathrm{b}}$ the points uniformly distributed in the bulk over a radius $\mathrm{R}_{\mathrm{b}}=0.7 \mathrm{R}$ ). The $\mathrm{Nr}_{\mathrm{s}}$ points near the free surface are clustered using the stretching function (s) due to Roberts and Eiseman (for further details see Fletcher [25]; for the present computations the stretching control parameters $\mathrm{P}=1$ and $\mathrm{Q}=2$ have been used). This corresponds to a grid stretching factor $\delta$ not constant with a maximum value $\left(\delta_{\max }\right)$ for the layer of points adjacent the free surface.

The code has been carefully validated through comparison with other results available in literature (for further details on the validation of the code and on the grid refinement analysis see Lappa et al. $[11,13,14])$. 


\subsection{Definition of the phase change zone thickness}

According to Brent et al. [24], in the case of an isothermal phase change (pure substance), the flow inside computational cells that are melting or freezing does not follow exactly the governing laws for flow in a mushy zone. In an isothermal phase-change system, the phase front is a well-defined line (in reality there will be a boundary layer on the solidification surface) and has no band or width associated with it as in the case of a mushy phase change.

Even if, in a isothermal phase change problem dealing with liquid metals, the mushy zone from a "physical point of view" is reduced to a very thin boundary layer, in a discretized calculation domain, anyway, a fixed grid solution technique "cannot capture" in detail this layer. The numerical technique in fact cannot handle thin boundary layers since, as pointed out by Brent et al. [24], the phase front will have a finite width associated with it, this width being at least that of a single computational cell. When the fixed-grid-enthalpy approach is employed, the only way to "capture" the "physics" of the boundary layer on the solidification surface, would be to reduce the width of the computational cells up to the real thickness of the layer where phase change occurs (few $\mu \mathrm{m}$ ). This is out the scope of the present work. For the case under investigation, moreover, (liquid column having initial length $\mathrm{L}=1[\mathrm{~cm}]$ ) this would require a prohibitive number of computational points along the axis of the liquid zone (of the order of $10^{3}-10^{4}$ ). Due to the fact that enormous speed and memory requirements are necessary for the direct numerical solution of the threedimensional Navier Stokes equations, for the present computations "only" 40 points have been used in axial direction (170 cpu hours of computations have been required for each simulation). The "thickness" of the mushy zone is strictly related to this number since its width corresponds to the $\Delta z$ coming out from the discretization of the liquid zone in axial direction (for the present computation, in agreement with the findings of Brent et al. [24], the thickness of the mushy zone corresponds to one/two layers of adjacent computational cells).

Moreover, as pointed out by Voller et al. [21], Bennon and Incropeira [22,23] and Brent et al. [24], it is desirable that the method chosen allows a smooth, gradual transition rather than a step change. For this reason the amplitude of the temperature range over which phase change occurs, has to be chosen in order to avoid step changes in the temperature fields (step changes in the momentum and energy equations source terms tend to retard convergence of their numerical solution, and 
sometimes lead to oscillations that may result in divergence), and at the same time in order to be consistent with the physics of the phenomenon under investigation: for the present case (phase change zone reduced to a thin boundary layer and 40 computational cells distributed in axial direction) a temperature range of $0.5[\mathrm{~K}]$ has been fixed; this choice has been proved to be a good compromise between the need to avoid step changes and the need to represent the phase change boundary layer with a number of computational cells in axial direction as low as possible. Preliminary computations have been carried out to show the negligible influence of the the temperature range chosen for the phase change zone on the problem under investigation.

According to Voller et al. [21], "the nature of the flow in the mushy region will influence its shape". This is true however when the solidification process is carried out in the case of high Prandtl number liquids (i.e. $\operatorname{Pr}>>1$, Voller et al. study the case of $\operatorname{Pr}=1000$ ). In these cases in fact, due to the low thermal diffusivity of the material (in the case of transparent substances usually $\left.\alpha=\mathrm{O}\left(10^{-4}\right)\left[\mathrm{cm}^{2} / \mathrm{s}\right]\right)$, in many situations the thickness of the mushy zone is very large. Due to this large thickness, the amount of convection "protruding" in the mushy zone is great and the flow inside this zone largely influences its shape. This however is not the case of a liquid metal $(\operatorname{Pr}<<1$, $\left.\alpha=\mathrm{O}\left(10^{-2}\right)\left[\mathrm{cm}^{2} / \mathrm{s}\right]\right)$. In the case of a low Prandtl number liquid, the mushy zone is reduced to a boundary layer (whose thickness corresponds to one/two layers of computational cells) and due to the "switch off" condition that drives velocities to zero in a so thin zone, the effect of the convection on the shape is very weak.

A test with a different size mushy region has been carried out to assess that the shape of this zone is not influenced by the temperature range chosen for the phase change zone. Since one may suspect that differences with respect to the computations carried out for an amplitude of the temperature range equal to $0.5[\mathrm{~K}]$ may arise when further reducing the size of the mushy region down to isothermal case, the amplitude of the temperature range has been reduced from 0.5 [K] to $0.25[\mathrm{~K}]$. The numerical simulation have shown that the influence of the amplitude of the temperature range (when this range is sufficiently low, close to the isothermal case) on the shape of the mushy zone is very weak. The size is reduced but the cystal/melt interface defined as the isoline at $\mathrm{T}=\mathrm{Tm}$ does not change its shape.

Regarding the effect of the thickness and curvature of the mushy zone on the flow, it should be pointed out moreover that the Marangoni flow is a "large scale flow" whose properties do not 
depend upon the local curvature of the boundary of the mushy zone and/or upon its thickness. A preliminary study has been carried out to assess that the large scale flow field does not depend upon the shape of the mushy region. At each instant the axial boundaries of the mushy region computed according to the temperature distribution have been replaced with average axial values obtained averaging the z-coordinate of the boundaries over their entire surface, thus making the mushy zone a "horizontal band". The results of this simulation show that the spatio-temporal behavior of the flow field (the structure of the field in the meridian plane and the azimuthal wave number of the instability and their modification as time passes) is the same as for the case of non-planar mushy zone.

\section{$\underline{3.3 \text { Model validation }}$}

The numerical technique has been validated through comparison with the recent experimental results of de Groh and Lindstrom [26] in the case of solidification of pure Succinonitrile. The article of de Groh and Lindstrom has been chosen since, as declared by the authors, their aim was to present interface shape and thermal data to be used to validate numerical models. Succinonitrile has been considered by de Groh and Lindstrom since it is transparent and the shape of the phase front to be compared with the numerical results can be determined with great accuracy from an experimental point of view. They conducted an experimental study of the crystal growth of Succinonitrile during solidification, melting and no-growth conditions using a horizontal Bridgman furnace and square glass ampoule $(15[\mathrm{~cm}]$ long, $0.8[\mathrm{~cm}]$ thick with a wall thickness of $0.1[\mathrm{~cm}])$. For use as input boundary conditions to numerical codes to be validated, thermal profiles on the outside of the ampoule were measured along its length and the shapes of the solid/liquid interface were quantitatively determined. The experiments were conducted with the furnace in a horizontal orientation, with an applied temperature difference of $75[\mathrm{~K}]$ and at furnace translation rates of 0 $[\mu \mathrm{m} / \mathrm{s}]$ (no growth), $40[\mu \mathrm{m} / \mathrm{s}]$ (solidifying) and $-40[\mu \mathrm{m} / \mathrm{s}]$ (melting). The second case has been considered here for comparison since it deals with the solidification process. In the numerical simulations the presence of the glass ampoule with a wall thickness of $0.1[\mathrm{~cm}]$ has been simulated in order to reproduce realistic conditions. A temperature range of $2[\mathrm{~K}]$ has been considered to model the zone over which phase change occurs. Central schemes have been used for the 
convective terms and 300x40 points for the computational grid. The comparison between numerical and experimental results is shown in Fig. 3.

\section{Results}

Liquid bridges of Gallium (see table I for the physical properties), having initial length $\mathrm{L}=1$ [cm] and two different initial aspect ratios $(A=1.0$ and $A=0.5)$, have been considered. 40 points have been collocated in the axial direction; in the radial and azimuthal directions $\mathrm{Nr}=22=11+11$ and $\mathrm{N} \varphi$ $=26$ in the case $A=1.0$ and $\mathrm{Nr}=26=13+13$ and $\mathrm{N} \varphi=30$ in the case $A=0.5$ (see Fig. 2). Each case required more than 170 cpu hours of computations on a Digital 433 au workstation.

For both the aspect ratios, a temperature difference of $4[\mathrm{~K}]$ has been considered (corresponding to a Marangoni number $\left.\mathrm{Ma}=\Delta \mathrm{T} \sigma_{\mathrm{T}} \mathrm{L} / \mu \alpha \cong 110\right)$.

For this value of the Marangoni number, the axisymmetric flow field is unstable and for both the aspect ratios a steady, three-dimensional flow regime is established after a certain time denoted by $t^{*}$. According to previous studies, the numerical results show that in this flow regime the position of the vortex core is displaced sinusoidally along the azimuthal perimeter of the liquid zone and the temperature field is characterized by sinusoidal distortions in azimuthal direction. Hereafter the differences between the temperature fields and the azimuthally averaged temperature distributions (referred to as temperature disturbances) will be reported in order to show clearly the azimuthal wave number .

For an initial aspect ratio $\mathrm{A}=1.0$, the steady and asymmetric supercritical Marangoni flow is characterized by an azimuthal wave number $\mathrm{m}=1$. Figs. $4 \mathrm{~b}$ and $4 \mathrm{c}$ show that in the generic crosssection orthogonal to the liquid bridge axis there are two azimuthal convective cells and two thermal spots on the liquid bridge surface (one cold and the other hot). The temperature contours in Fig. 4d show a lower temperature inner region whose shape is circular but whose position is eccentric to the geometrical axis of symmetry of the liquid bridge. In this case the supercritical flow appears as an inclined toroidal vortex. In each meridian plane of the liquid bridge there are two asymmetrical vortex cells (one of the two vortex cells in the section prevails over the other and is extended along the whole axial plane of the bridge, for instance see Fig. 4a). 
For $\mathrm{t}>\mathrm{t}^{*}$, the temperature on the cold disk is reduced with a ramp of $0.1[\mathrm{~K} / \mathrm{s}]$ and the crystallization front advances, as shown in Figures 5-9. The temperature interval chosen to represent the range over which phase change occurs $\left(2 \Delta \mathrm{T}_{\mathrm{m}}\right)$ has been fixed to $0.5[\mathrm{~K}]$.

Due to the azimuthal distortion of the thermo-fluid-dynamic field, the shape of the crystal is not axisymmetric. This is shown clearly in Fig. 5a and in Fig. 10a for $\mathrm{A}_{\mathrm{i}}=0.885\left(\mathrm{Ma}_{\mathrm{i}}=100\right)$. In Fig. 5a the distortion of the boundaries of the transition zone is shown in relationship to the asymmetric flow field structure in the meridian plane $\varphi=-\pi / 6$. The distortion of the boundaries of the mushy zone is more pronounced on the left side of the liquid zone where the strongest vortex cell is present. Due to this distortion, the thickness of the transition zone is not uniform in the meridian plane; the thickness is larger in the right side. In Fig. 10a the temperature iso-surface corresponding to $\mathrm{T}=\mathrm{T}_{\mathrm{m}}$ (average crystal $/$ melt interface) is shown. The shape of the surface is convex. Due to the steady Marangoni instability, it is azimuthally distorted and has an axial minimum at $\varphi=-\pi / 6$. The corresponding surface temperature disturbance distribution (Fig. 5c) shows that the azimuthal wave number is still $\mathrm{m}=1$ since only two temperature spots are present. However the hot spot is more extended in azimuthal direction with respect to the cold one. This situation is different compared to that observed at $\mathrm{t}=\mathrm{t}^{*}$, where the two spots have exactly the same azimuthal extension. This behaviour suggests that the azimuthal organization of the flow has been influenced by the presence of the crystal.

For $\mathrm{A}_{\mathrm{i}}=0.72\left(\mathrm{Ma}_{\mathrm{i}}=82\right.$, Fig. $\left.6 \mathrm{c}\right)$, the spots on the free surface become four (two hot and two cold). This indicates that the azimuthal wave number is changed from $m=1$ to $m=2$, due to the solidification process. Four convective cells appear in the section as shown in Fig. 6b; the inner region in this case is not circular but approximately elliptic with centre corresponding to the geometrical axis of symmetry of the liquid bridge (see Fig. 6d).

According to previous results (Lappa et al. [11]), for even azimuthal wave numbers, the flow field structure is on the whole three-dimensional and depends on the azimuthal co-ordinate, but in each meridian plane the velocity and temperature are symmetric (for instance see Fig. 6a). For this reason the azimuthal distortion of the mushy region boundaries due to the Marangoni flow instability cannot be highlighted in the generic meridian plane. Fig. 10b shows that the shape of the crystal/melt interface is azimuthally distorted and has two axial minima $(\varphi=5 \pi / 6$ and $\varphi=-\pi / 6)$ and two maxima $(\varphi=\pi / 3$ and $\varphi=4 \pi / 3)$ in azimuthal direction. 
For $\mathrm{A}_{\mathrm{i}}=0.5\left(\mathrm{Ma}_{\mathrm{i}}=57\right.$, Figs. $\left.7 \mathrm{a}-7 \mathrm{~d}\right)$, the azimuthal wave number is $\mathrm{m}=2$, as for $\mathrm{A}_{\mathrm{i}}=0.72$; Fig. $7 \mathrm{c}$ shows that in this case the amplitude of the temperature disturbances on the free surface is lower.

When the melt aspect ratio is further decreased $\left(\mathrm{A}_{\mathrm{i}}<0.5\right)$ due to the solidification process, the thickness of the mushy region decreases (see Figs. 8a, 9a) with a linear dependence on $\mathrm{A}_{\mathrm{i}}$. The Marangoni flow becomes more and more confined near the free surface (the radial extension of the vortex cells in the meridian plane becomes approximately equal to the axial extension of the liquid zone held between the crystal surface and the hot supporting disk, see Fig 8a and 9a). For this reason the isolines of the temperature field in the inner part (near the axis) of the bridge are no longer convex and become concave. Correspondingly, the iso-surface $\mathrm{T}=\mathrm{T}_{\mathrm{m}}$ for $\mathrm{A}_{\mathrm{i}}=0.33$ (Fig. 10d) shows a concave shape in the inner part (near the axis) and a convex shape near the free surface. The azimuthal distortion of the shape is more pronounced in the inner part whereas an axisymmetric behaviour can be observed far from the axis. This tendency is confirmed by the fact that the temperature disturbances are negligible on the free surface (Fig. 8c) whereas the temperature distribution is not symmetric in the inner part of the generic cross-section orthogonal to the axis (Fig. 8d).

For $\mathrm{A}_{\mathrm{i}}=0.28\left(\mathrm{Ma}_{\mathrm{i}}=32\right)$ full stabilization of the flow field is reached (subcritical bifurcation). The azimuthal disturbances vanish (Figs. $8 \mathrm{~b}$ and $8 \mathrm{c}$ ) and the shape of the crystal is axisymmetric (Fig. 10e).

For an initial aspect ratio $\mathrm{A}=0.5$, at the initial time $\mathrm{t}=\mathrm{t}^{*}$ the supercritical Marangoni flow is still steady and asymmetric (as for $\mathrm{A}=1.0$ ) but it is characterized by azimuthal wave number $\mathrm{m}=2$. There are four convective cells in the cross-section orthogonal to the axis and four temperature spots on the free surface (two hot and two cold, see Figs. 11). In the generic meridian plane the flow field is symmetric but with different radial and axial organization with respect to the other planes corresponding to other values of the azimuthal coordinate $\varphi$ (Figs. 11a and 11b).

When solidification occurs (ramp of $0.1[\mathrm{~K} / \mathrm{s}]$ ), for $0.24<\mathrm{A}_{\mathrm{i}} \leq 0.5$, the azimuthal wave number $\mathrm{m}=2$ is stable. For $\mathrm{A}_{\mathrm{i}}=0.24\left(\mathrm{Ma}_{\mathrm{i}}=55\right.$, see Figs. 12) however, the hot spots on the free surface show a doubling mechanism similar to that described in the case $m=1(A=1.0)$. Each hot spots seems to double increasing its azimuthal extension (see Fig. 12c). The crystal/melt interface is concave near the axis and convex near the free surface and the azimuthal distortion is more pronounced in the inner part (Fig. 14a) as described for the cases $A=1.0$ and $A_{i}<0.5$. 
For $\mathrm{A}_{\mathrm{i}}=0.18\left(\mathrm{Ma}_{\mathrm{i}}=41\right)$, a mode $\mathrm{m}=4$ appears. There are, respectively, eight vortex cells and surface spots, as shown in Figs. 13b and 13c. Moreover a more complicated non eccentric pattern appears in the temperature field of the cross-section. For $m=4$ the inner region has the form of a quadrangle (Fig. 13d). Correspondingly the crystal/melt interface shape shows four axial maxima and four minima $(\varphi=3 \pi / 7, \varphi=\pi-\pi / 14, \varphi=10 \pi / 7, \varphi=-\pi / 14)$ in azimuthal direction (Fig. 14b).

For $\mathrm{A}_{\mathrm{i}}=0.135\left(\mathrm{Ma}_{\mathrm{i}}=31\right)$ full stabilization of the thermo-fluid-dynamic field occurs (see Fig. 14c).

\section{Discussion}

The azimuthal distortion of the shape of the crystal/melt interface is explained on the basis of the toroidal structure of the supercritical flow field and of the temperature distribution.

The temperature disturbances have m maxima (minima) in azimuthal direction (as shown by the Figures $4 \mathrm{c}$ to $8 \mathrm{c})$. The nodes of the geometrical patterns given by the isolines of the temperature field in the cross-section orthogonal to the axis simply correspond to these extrema in the temperature field. For $m=1$ the geometrical pattern is an eccentric circle (Fig. $4 d$ ) since there are a minimun and a maximum in the temperature field (the circle corresponds to the minimum). For $\mathrm{m}=2$ the geometrical pattern is an ellipse since there are two minima and two maxima (in Fig. $6 \mathrm{~d}$ the main axes of the ellipse connect respectively the axis of symmetry of the bridge with the positions corresponding to a minimum and to a maximum of the temperature disturbance distribution). For $\mathrm{m}=4$ (see e.g. Fig. 13d) the geometrical pattern is a quadrangle since the temperature disturbances have four maxima.

The radial position of the nodal lines of the temperature perturbations also corresponds to the position of the vortex centerline after the bifurcation. The present results show that the position of the vortex core is deformed and in particular displaced sinusoidally along the perimeter of the toroidal convection roll. This is due to the occurrence of the additional convective cells (strictly related with the Marangoni flow instability) in the sections orthogonal to the bridge axis (shown in Figs. $5 \mathrm{~b}$ to $9 \mathrm{~b}$ and $12 \mathrm{~b}$ and $13 \mathrm{~b}$ ) convecting fluid in azimuthal direction.

The results on the crystal/melt interface shape show that this surface describes in azimuthal direction a sine curve having $\mathrm{m}$ maxima and $\mathrm{m}$ minima in the $\mathrm{z}$-direction. This distortion can be 
simply related to the sinusoidal spatial deformation of the temperature field, due to the distorted spatial displacement of the vortex core in the supercritical state.

Regarding the occurrence of different values of the azimuthal wave numbers during the solidification process $(\mathrm{m}=1$ and $\mathrm{m}=2$ for $\mathrm{A}=1.0$, and $\mathrm{m}=2$ and $\mathrm{m}=4$ for $\mathrm{A}=0.5)$, the explanation for this behaviour can be found in the relation between the nature of the three-dimensional disturbances and the geometry of the liquid zone.

For cylindrical liquid bridges previous numerical computations ([7-11]) have shown that the flow structure of the supercritical state depends on the value of the aspect ratio of the liquid bridge. The lower the aspect ratio, the higher the azimuthal wave number m resulting in the more complex flow organization. It is known that discrete wavenumbers of disturbances are selected out of the full spectrum of disturbances because the convection roll is closed in a special zone geometry. When the instability is hydrodynamic in nature (i.e. it does not depend on the temperature field, that simply acts as a driving force for the velocity field), the selection rule is given simply by the constraint that the azimuthal wavelength must be an aliquot of the toroidal vortex core circumference and by the fact that the convection roll is limited axially by the presence of the sidewalls (see Lappa et al. [11]). According to this theory, for the problem under investigation, the critical wave number is related to the axial length (distance between the the hot disk and the crystal/melt interface) and to the diameter of the liquid zone, i.e. in the present case it scales with the parameter $\mathrm{A}_{\mathrm{i}}=\mathrm{L}_{\mathrm{i}} / \mathrm{D}$. The present numerical results show a simple correlation between the melt aspect ratio and the azimuthal wave number of the instability:

$\mathrm{mA}_{\mathrm{i}} \cong 1$

The stabilization of the flow field, observed for both the cases here considered $(A=1.0$ and $A=0.5)$ when the melt aspect ratio becomes less than a certain value, is explained by the simultaneous reduction of the instantaneous Marangoni number and of the melt aspect ratio. There are in fact two different effects leading to the stabilization of the flow field. For a fixed aspect ratio, decreasing the Marangoni number, subcritical conditions prevail. On the other hand, as found by Lappa et al.[11], for $\mathrm{A}<0.5$, lower is the aspect ratio, higher should be the critical Marangoni number. 
The stabilization of the flow field can be explained also focusing the attention directly on the nature of the instability. As highlighted in reference [4], for low Prandtl number liquids, the bifurcation of the flow field to a steady three-dimensional configuration is directly related to the inertial instability of the shear layer below the free surface; the instability takes energy from the radial gradient of the axial velocity, i.e. the axial share. Marangoni forces driving the flow field induce a strong axial shear flow. For this reason the bifurcation is favored by increasing the Marangoni number (driving action) and opposed by the presence of walls (where "no-slip" prevails). For the specific problem under investigation, when the aspect ratio decreases, the ratio of the free surface area $\mathrm{S}_{1}=\pi \mathrm{DL}_{\mathrm{i}}$ (where the "driving force" is present) divided by the end walls area $\mathrm{S}_{\mathrm{W}}=\pi \mathrm{D}^{2 / 2}$ (where "no-slip" prevails), $\mathrm{S}_{\mathrm{l}} / \mathrm{S}_{\mathrm{w}}=2 \mathrm{~A}_{\mathrm{i}}$ also decreases, increasing viscous effects; moreover the decrease of the instantaneous value of the Marangoni number decreases the Marangoni stress on the free liquid surface. The simultaneous increase of the viscous effects and decrease of the Marangoni stress stabilize the flow field.

\section{Conclusions}

Marangoni flow instability during solidification in half zone liquid bridges in zero g conditions has been analyzed by direct solution of the three-dimensional and time-dependent non-linear equations. A single region (continuum) formulation (enthalpy-porosity technique) has been adopted to model the phase change problem, solving the coupled temperature and velocity fields over the whole computational domain, including the solid and liquid phases.

The enthalpy-porosity approach has been extended to the case of problems with free surface. The typical "stress-balance" Marangoni flow boundary conditions have been modified in order to take into account the phase change phenomenon. This is an original contribution to the enthalpy-porosity technique.

For the first time that this approach has been applied to the case of a floating zone. The influence of the azimuthally asymmetric convective field on the shape of the crystal/melt interface during the solidification process and the relationship among the azimuthal wave number of the instability and the relative position of the solid/melt interface at each instant have been investigated. 
The numerical results show that the crystal/melt interface is distorted in azimuthal direction due to the sinusoidal spatial deformation of the temperature field, related to the spatial displacement of the vortex core caused by the instability of the Marangoni flow.

Different values of the azimuthal wave numbers were found during the solidification process. This result is explained on the basis of the relation between the typology of the azimuthal disturbances and the structure of the fluid-dynamic field. A generalized law is found to correlate the azimuthal wave number of the instability to the extension of the melt zone.

Until now, all the numerical investigations dealing with the analysis of the relationship between the azimuthal wave number and the aspect ratio of the liquid zone, have been obtained in the case of "static" liquid bridges (fixed distance between the supporting disks and no phase change). The results presented in this paper represent a first contribution to the modelling and understanding of complex phenomena caused by fluid flow and simultaneous solidification during floating zone crystal growth.

A novel research programme is being proposed for the early utilization phase of the International Space Station (ISS) to analyze in detail Marangoni flow in solidifying liquid bridges, using materials with low mwlting point (e.g. Succinonitrile) that are transparent in the liquid state.

\section{Acknowledgments}

The authors would like to thank the Referees for their constructive comments.

\section{References}

[1] K. W. Benz, "Factors controlling crystal perfections during growth under microgravity", Proceedings VIIth European Symposium on Materials and Fluid Sciences in Microgravity Oxford (United Kingdom), 10/15 September, ESA SP 295, 59, (1990)

[2] F. Preisser, D. Schwabe, A. Scharmann, "Steady and oscillatory thermocapillary convection in liquid columns with free cylindrical surface", J.Fluid Mech. 126, 545, (1983) .

[3] H. C. Kuhlmann, H. J. Rath, "Hydrodinamic instabilities in cylindrical thermocapillary liquid bridges", J.Fluid Mech. , 247, 247, (1993).

[4] M. Wanschura, V. Shevtsova, H.C. Kuhlmann, H.J. Rath, Convective instability mechanism in thermocapillary liquid bridges, Phys. Fluids, 5, 912, (1995). 
[5] R. Rupp, G. Muller, G. Neumann, "Three dimensional time dependent modelling of the Marangoni convection in zone melting configurations for GaAs", Journal of Crystal growth, 97, 34, (1989).

[6] M. Levenstam, G. Amberg, "Hydrodynamical instabilities of thermocapillary flow in a halfzone", J.Fluid Mech., 297, 357, (1995).

[7] N. Imaishi, S. Yasushiro, T. Sato, S. Yoda, "Numerical simulation of three dimensional oscillatory flow in half zone bridges of low Pr fluid", Proceedings 292 of the 4th JSME-KSME Thermal Engineering Conference - October 1-6, 2000, Kobe, Japan, 272

[8] S. Yasushiro, T. Sato, N. Imaishi, S.Yoda "Three dimensional Marangoni flow in liquid bridge of low Pr fluid", Space Forum, 6, 39 (2000)

[9] J. Leypoldt, H.C. Kuhlmann, H.J. Rath, "Three-dimensional numerical simulation fo thermocapillary flows in cylindrical liquid bridges", J. Fluid Mech, 414, 285, (2000)

[10] M. Lappa , R. Savino, "Parallel solution of three-dimensional Marangoni flow in liquid bridges", Int. J. Num. Meth. Fluids, 31, 911, (1999)

[11] M. Lappa, R. Savino and R. Monti, "Three-dimensional numerical simulation of Marangoni instabilities in non-cylindrical liquid bridges in microgravity"; Int. J. of Heat and Mass Transfer, 44 Issue 10, 1983, (2001)

[12] V.M. Shevtsova, D.E. Melnikov, "Influence of variable viscosity on convective flow in liquid bridges. 3-D simulations of ground based experiments", 1-st Symp. on Microgravity Research and Applications in Physical Science and Biotechnology, Sorrento, Italy, Sept. 2000

[13] M. Lappa, R. Savino and R. Monti; "Three-dimensional numerical simulation of Marangoni instabilities in liquid bridges: influence of geometrical aspect ratio", Int. J. Num. Meth. Fluids, 36 (1), 53, (2001)..

[14] M. Lappa, R. Savino and R. Monti; "Influence of buoyancy forces on Marangoni flow instabilities in liquid bridges"; Int. J. Num. Meth. Heat Fluid Flow, 10, Issue 7, 721, (2000)

[15] S. Nakamura, K. Kakimoto, T. Hibiya, (1995), "Convection visualization and temperature fluctuation measurement in a molten silicon column", Materials and fluids under low gravity (Springer), Proceedings of the IXth European Symposium on Gravity-Dependent Phenomena in Physical sciences Held at Berlin, Germany, 2-5 May 1995

[16] S. Nakamura, T. Hibiya, K. Kakimoto, N. Imaishi, S-i Nishizawa, A. Hirata, K. Mukai, S-i Yoda, T. S. Morita, "Temperature fluctuations of the Marangoni flow in a liquid bridge of molten silicon under microgravity on board the TR-I4 rocket", Journal of Crystal Growth 186, 85, (1998).

[17] S. Nakamura, T. Hibiya, N. Imaishi, S. Yoda, "Observation of Marangoni convection in a halfzone silicon melt", 32nd Scientific Assembly at COSPAR 1998 Nagoya - G0.1-0059

[18] T. Hibiya, S. Nakamura, K. Mukai, Z-G Niu, N. Imaishi, S-i Nishizawa, S-i Yoda, M. Koyama, "Interfacial phenomena of molten silicon: Marangoni flow and surface tension", Phil. Trans. R. Soc. Lond. A 356, 899, (1998).

[19] T. Hibiya, S. Nakamura, N. Imaishi, K. Mukai, K. Onuma, P. Dold, A. Croll, K-W Benz, S-i Yoda, "Marangoni flow of Si melt: microgravity experiments and perspective", J. Jpn. Soc. 
Microgravity Appl. Supplement, Proc. Joint 1st Pan-Pacific Basin Workshop and 4th Japan-China Workshop on Microgravity Sciences (1998).

[20] S. Nakamura, T. Hibiya, N. Imaishi, S.Yoda, T.Nakamura and M. Koyama, "Flow visualization and temperature measurements in a Half-Zone silicon bridge on board the TR-IA-6 Rocket", Microgravity sci. technol. XI/1 (1999)

[21] V. R. Voller, C. Prakash, "A fixed grid numerical modelling methodology for convectiondiffusion mushy region phase-change problems", Int. J. Heat Mass Transfer, 30 (8), 1709, (1987).

[22] W.D. Bennon, F.P. Incropera, "A continuum model for momentum, heat and species transport in binary solid-liquid phase change systems-I. Model formulation", Int.J.Heat Mass Transfer., 30 (10), 2161, (1987).

[23] W.D. Bennon, F.P. Incropera, "A continuum model for momentum, heat and species transport in binary solid-liquid phase change systems-II. Application to solidification in a rectangular cavity", Int.J.Heat Mass Transfer., 30 (10), 2171, (1987).

[24] A. D. Brent, V.R. Voller, J. Reid, "Enthalpy-porosity technique for modelling convectiondiffusion phase change: application to the melting of a pure metal", Numerical Heat Transfer, 13, 297, (1988).

[25] C.A.J. Fletcher, "Computational techniques for fluid-dynamics", (Springer Verlag, Berlin, 1991)

[26] H. C. de Groh III, T. Lindstrom, "Interface shape and convection during solidification and melting of succinonitrile", NASA TM 106487 (1994). 


\begin{tabular}{||c|c||}
\hline density $\rho\left[\mathrm{gr} / \mathrm{cm}^{3}\right]$ & 6.10 \\
\hline thermal diffusivity $\alpha\left[\mathrm{cm}^{2} / \mathrm{s}\right]$ & $1.6610^{-1}$ \\
\hline kinematic viscosity $\nu\left[\mathrm{cm}^{2} / \mathrm{s}\right]$ & $3.4410^{-3}$ \\
\hline dynamic viscosity $\mu[\mathrm{g} / \mathrm{cms}]$ & $2.1210^{-2}$ \\
\hline specific heat coefficient $\mathrm{Cp}[\mathrm{J} / \mathrm{gK}]$ & 0.40794 \\
\hline latent heat of fusion $\mathrm{L}_{\mathrm{m}}[\mathrm{J} / \mathrm{gK}]$ & 80 \\
\hline melting temperature $\mathrm{T}_{\mathrm{m}}[\mathrm{K}]$ & 302.91 \\
\hline$\sigma_{\mathrm{T}}[$ dyne $/ \mathrm{cmK}]$ & $110^{-1}$ \\
\hline
\end{tabular}

Table I: Physical properties of Gallium 


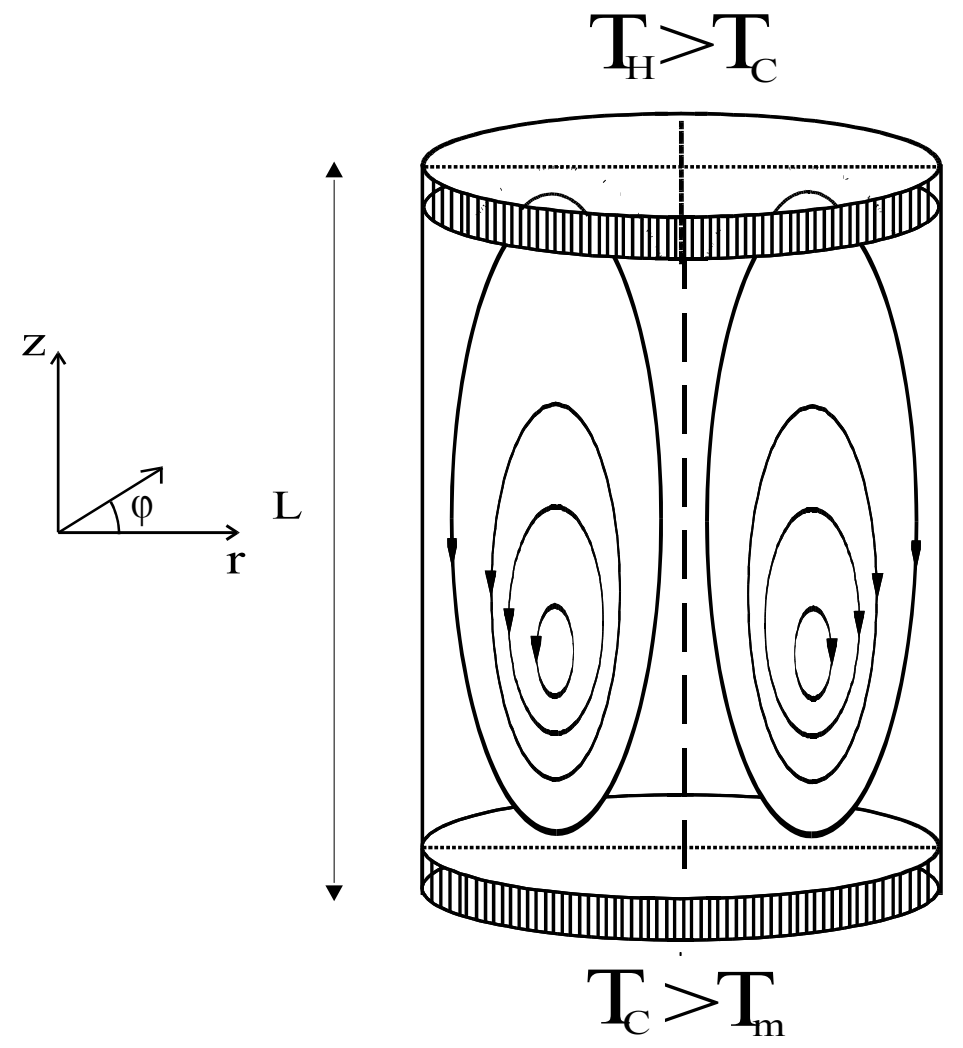

(a)

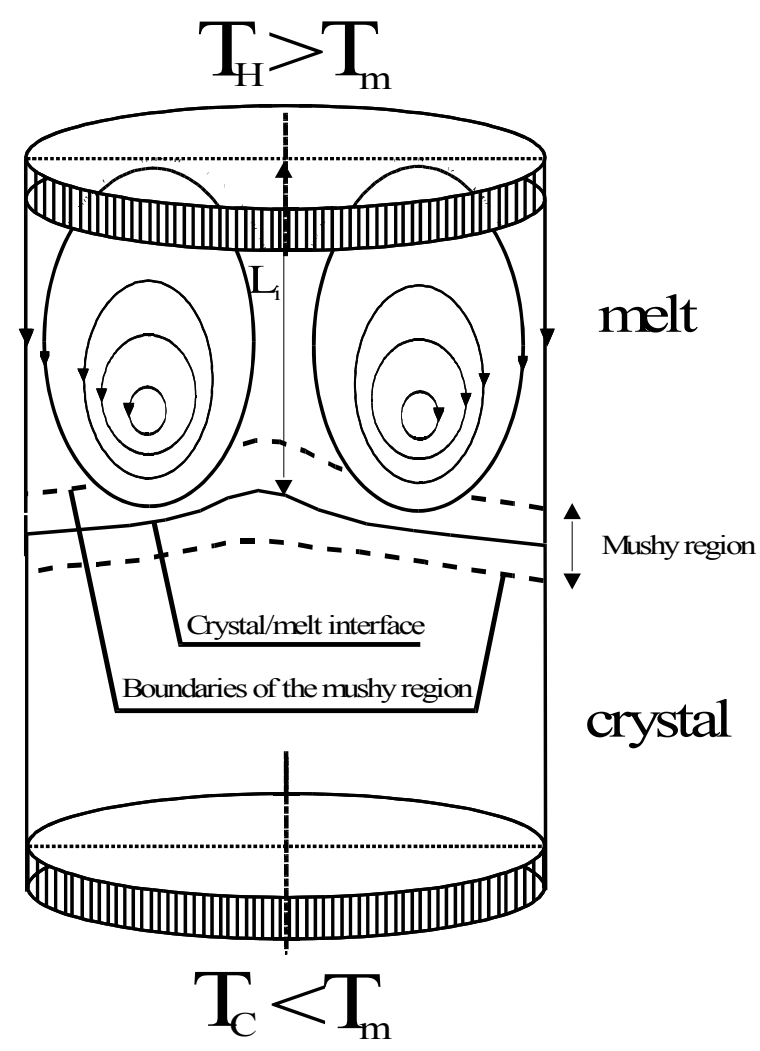

(b)

Figs.1: sketch of the liquid bridge 


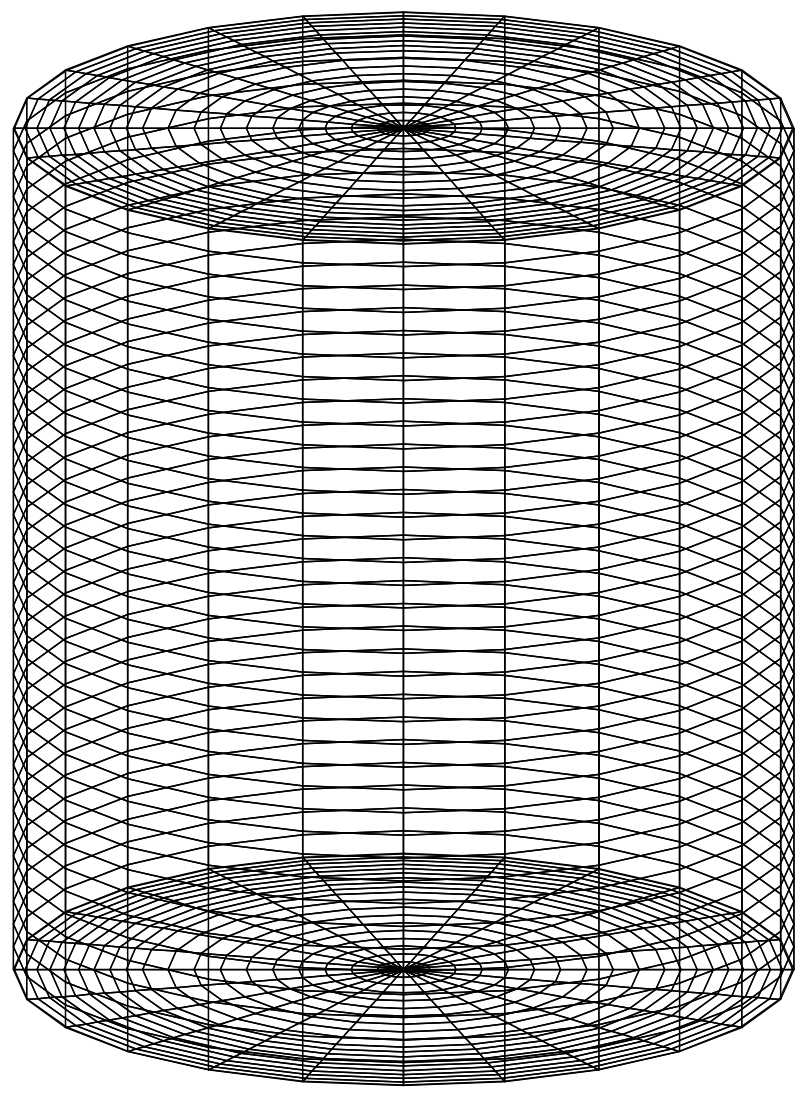

Fig.2

Computational grid 


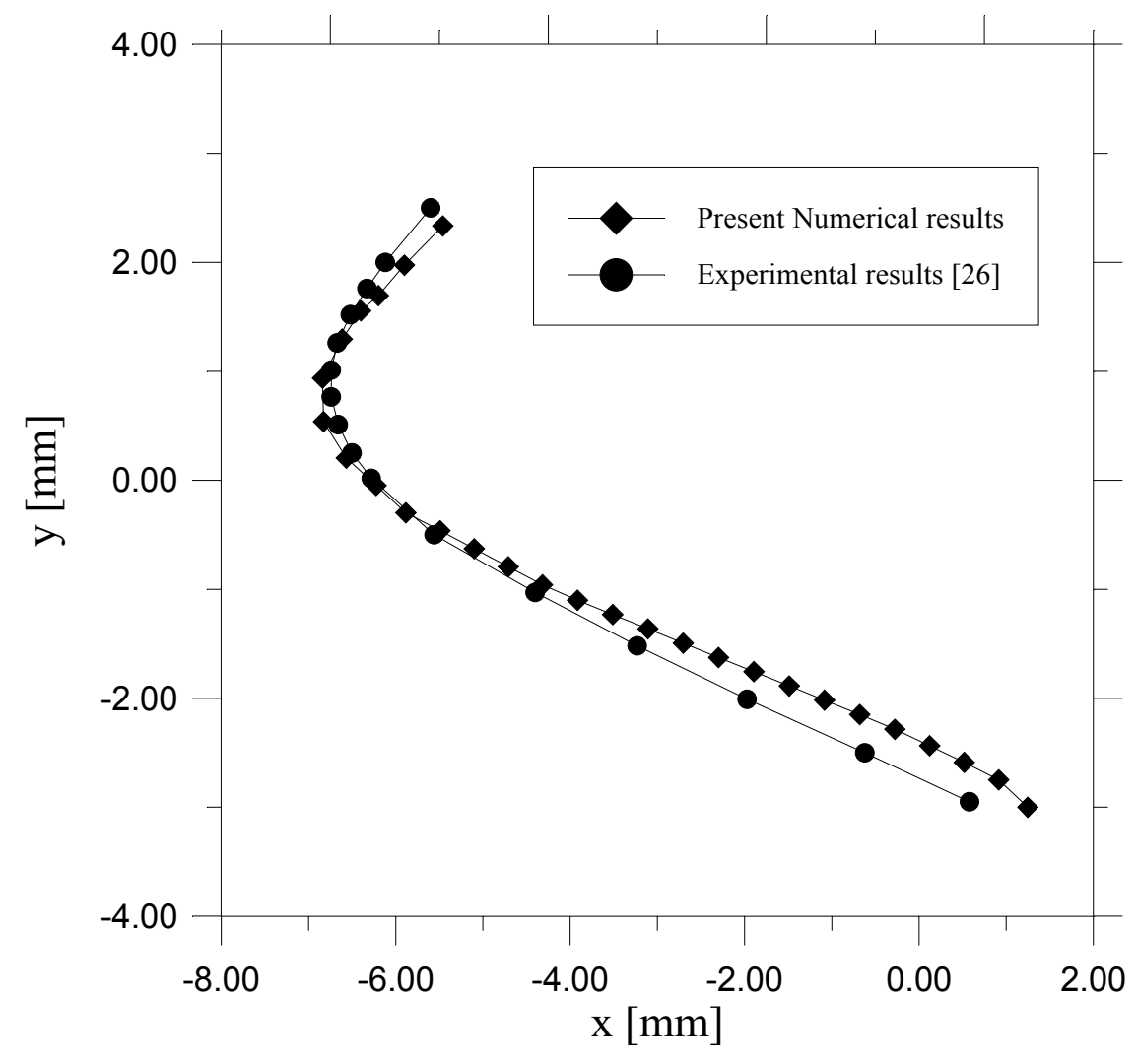

Fig.3: validation of the phase change technique - comparison between experimental and numerical results 


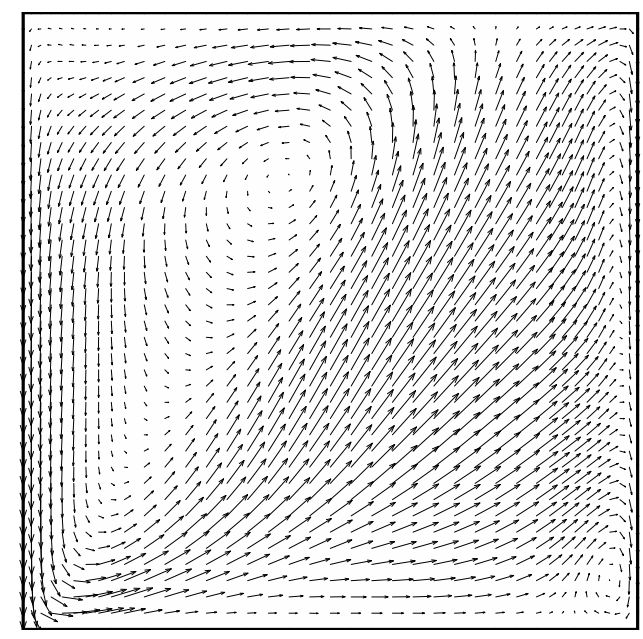

Fig.4a: Flow field in the meridian plane $\varphi=-\pi / 6\left(\mathrm{~A}=1.0, \mathrm{Ma}=110, \mathrm{t}=\mathrm{t}^{*}\right)$

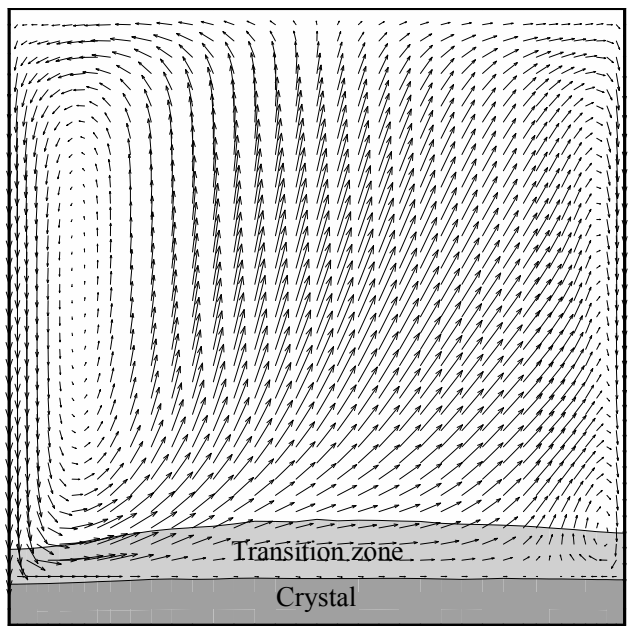

Fig.5a: Flow field in the meridian plane $\varphi=-\pi / 6\left(\mathrm{~A}_{\mathrm{i}}=0.885, \mathrm{Ma}_{\mathrm{i}}=100, \mathrm{t}-\mathrm{t}^{*}=2.6\right)$

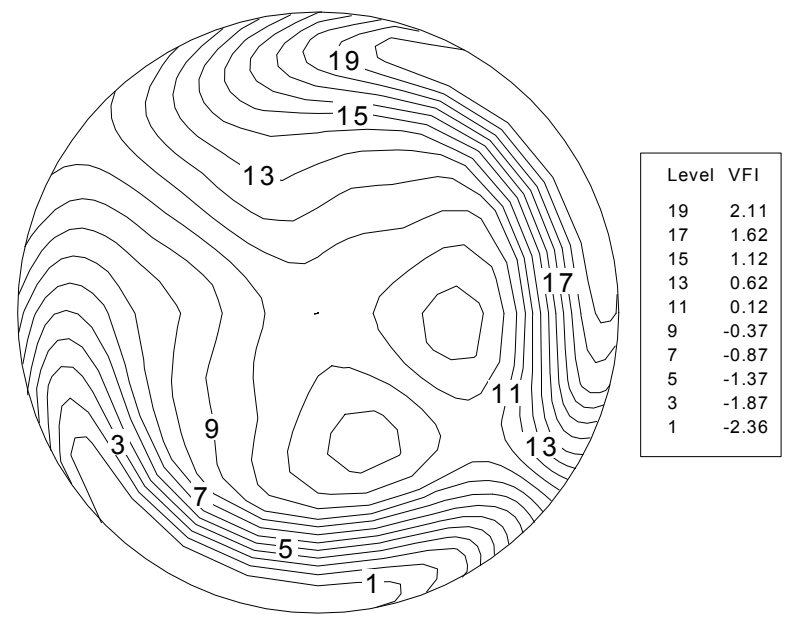

Fig. 4b: azimuthal velocity distribution in the section $\mathrm{z}=0.75\left(\mathrm{~A}=1.0, \mathrm{Ma}=110, \mathrm{t}=\mathrm{t}^{*}\right)$

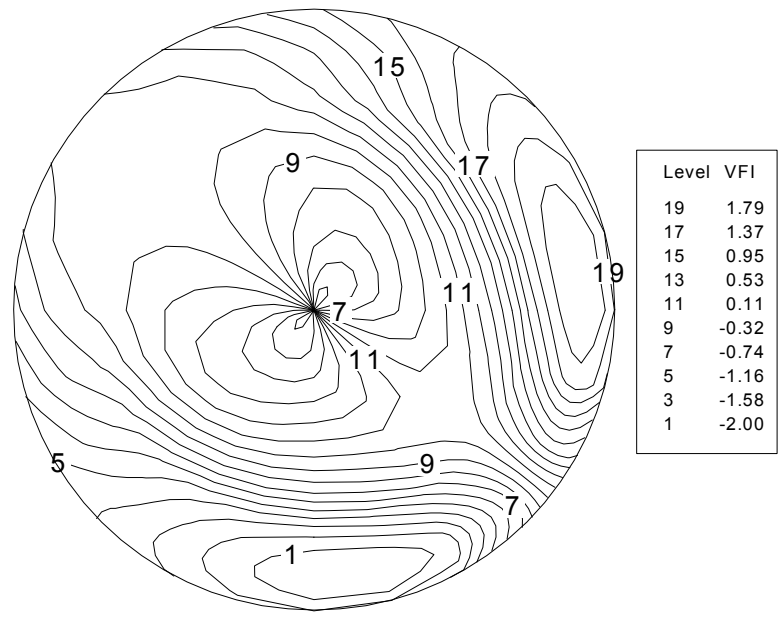

Fig. 5b: azimuthal velocity distribution $\mathrm{z}=0.75\left(\mathrm{~A}_{\mathrm{i}}=0.885, \mathrm{Ma}_{\mathrm{i}}=100, \mathrm{t}-\mathrm{t} *=2.6\right)$ 


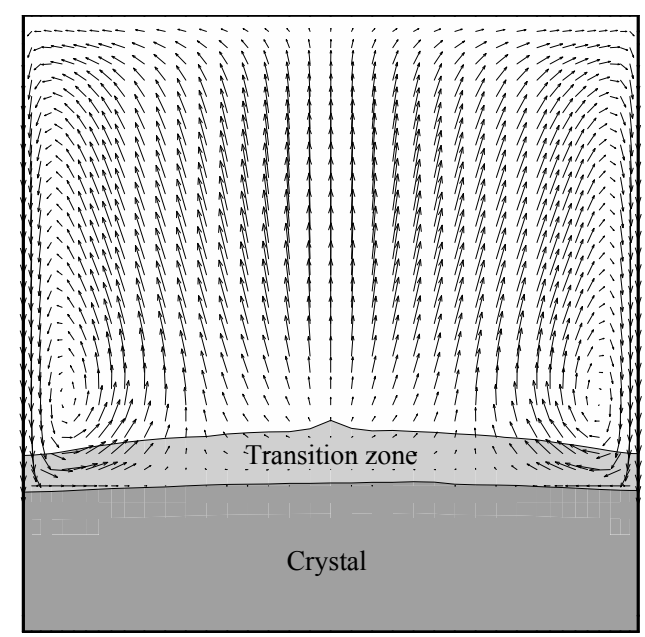

Fig.6a: Flow field in the meridian plane $\varphi=-\pi / 6\left(\mathrm{~A}_{\mathrm{i}}=0.72, \mathrm{Ma}_{\mathrm{i}}=82, \mathrm{t}-\mathrm{t} *=6.2\right)$

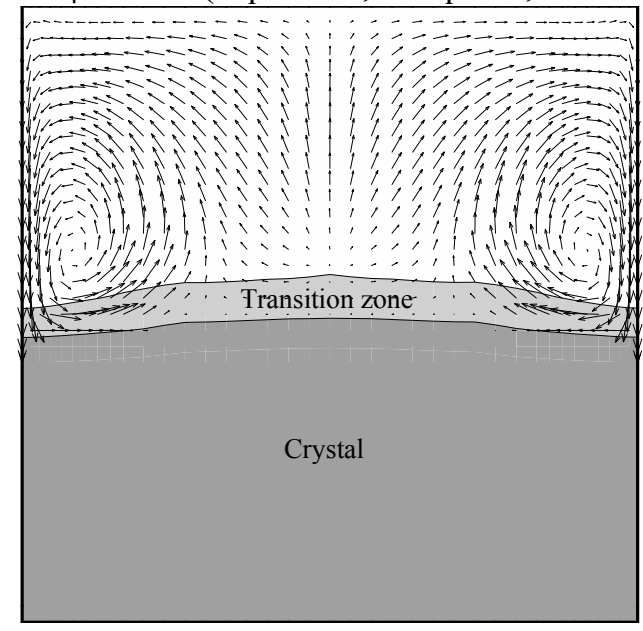

Fig.7a: Flow field in the meridian plane $\varphi=-\pi / 6\left(\mathrm{~A}_{\mathrm{i}}=0.50, \mathrm{Ma}_{\mathrm{i}}=57, \mathrm{t}-\mathrm{t}^{*}=11.9\right)$

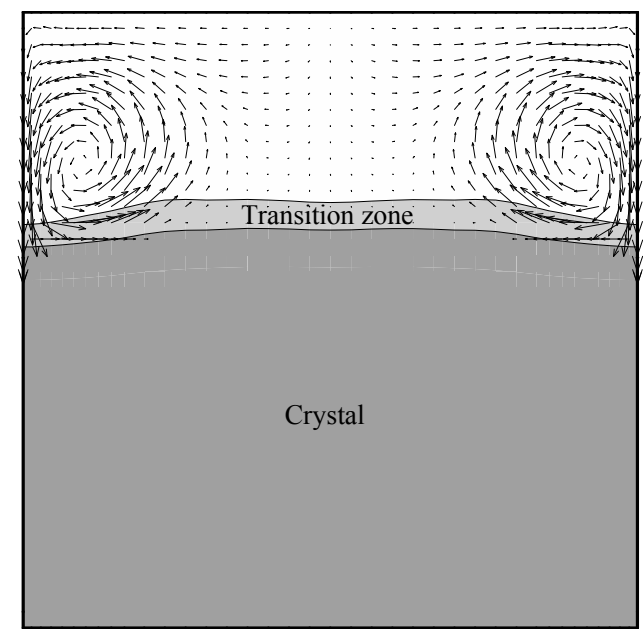

Fig.8a: Flow field in the meridian plane $\varphi=-\pi / 6\left(\mathrm{~A}_{\mathrm{i}}=0.33, \mathrm{Ma}_{\mathrm{i}}=37, \mathrm{t}-\mathrm{t}^{*}=17.5\right)$

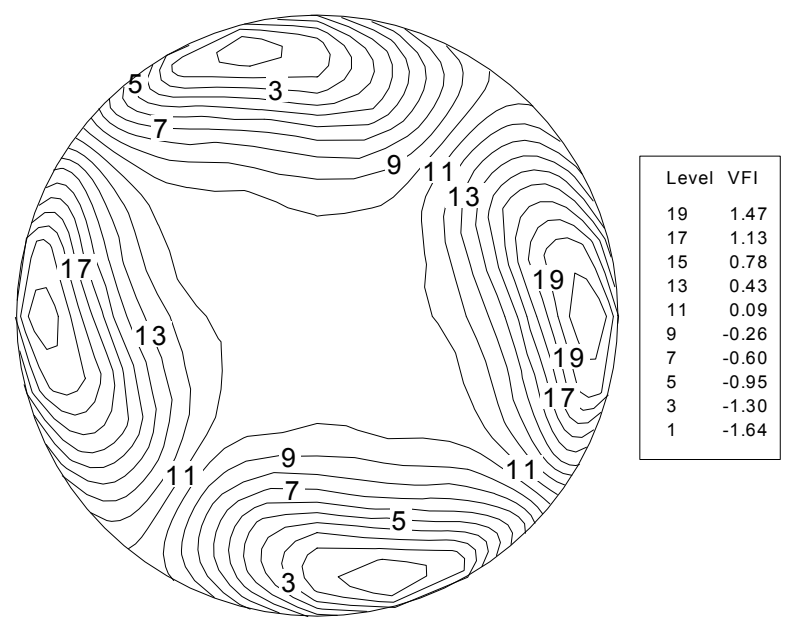

Fig. 6b: azimuthal velocity distribution $\mathrm{z}=0.75\left(\mathrm{~A}_{\mathrm{i}}=0.72, \mathrm{Ma}_{\mathrm{i}}=82, \mathrm{t}-\mathrm{t}^{*}=6.2\right)$

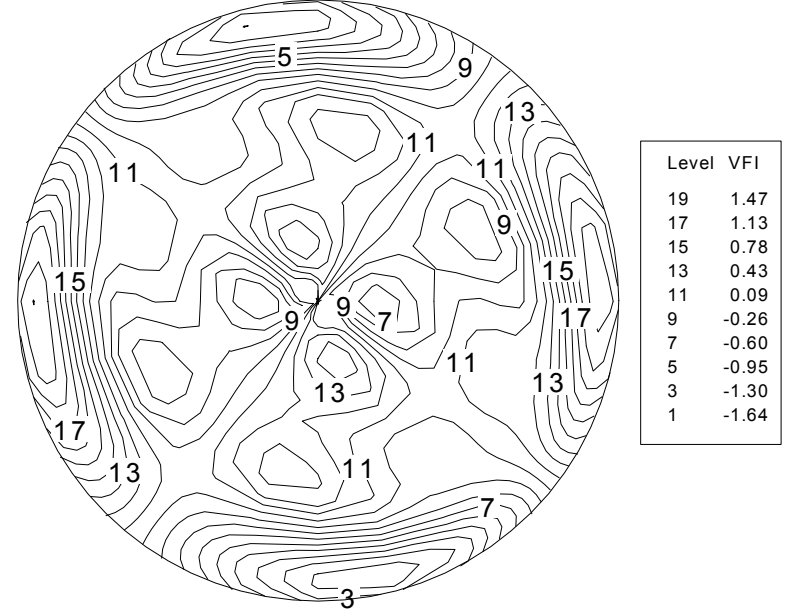

Fig. 7b: azimuthal velocity distribution $\mathrm{z}=0.75\left(\mathrm{~A}_{\mathrm{i}}=0.50, \mathrm{Ma}_{\mathrm{i}}=57, \mathrm{t}-\mathrm{t}^{*}=11.9\right)$

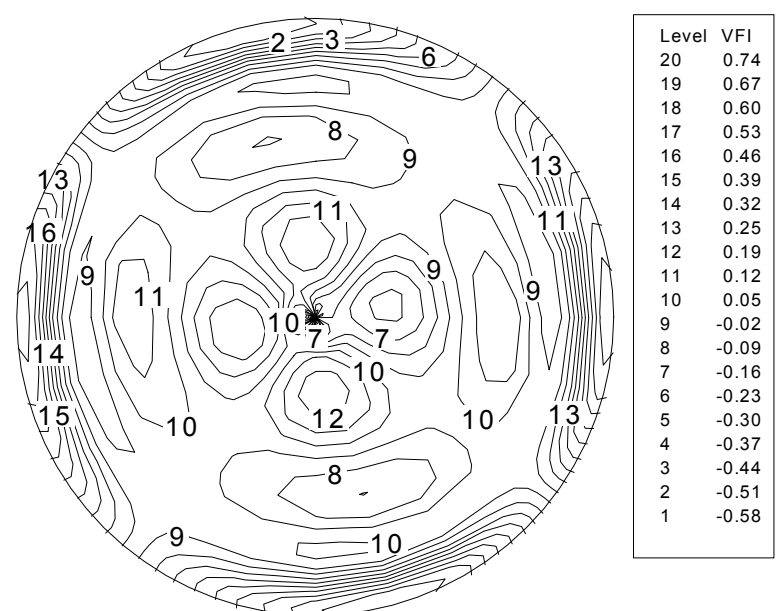

Fig. 8b: azimuthal velocity distribution $\mathrm{z}=0.75\left(\mathrm{~A}_{\mathrm{i}}=0.33, \mathrm{Ma}_{\mathrm{i}}=37, \mathrm{t}-\mathrm{t}^{*}=17.5\right)$ 


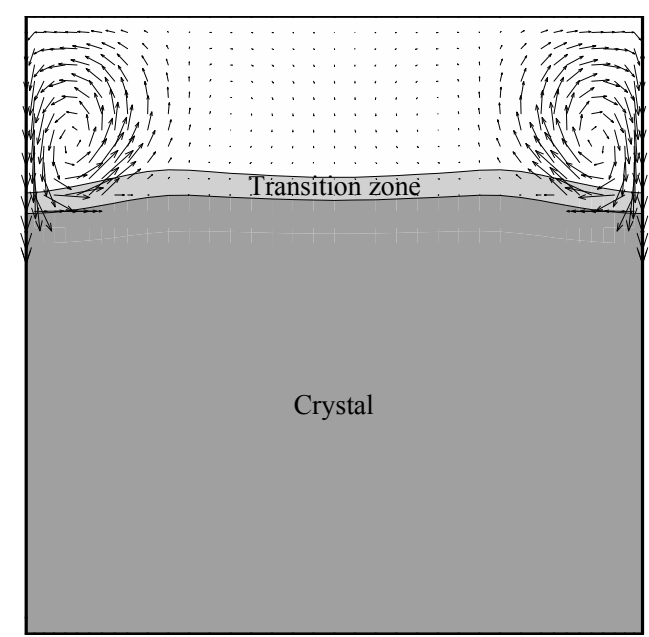

Fig.9a: Flow field in the meridian plane $\varphi=-\pi / 6\left(\mathrm{~A}_{\mathrm{i}}=0.28, \mathrm{Ma}_{\mathrm{i}}=32, \mathrm{t}-\mathrm{t}^{*}=20.6\right)$

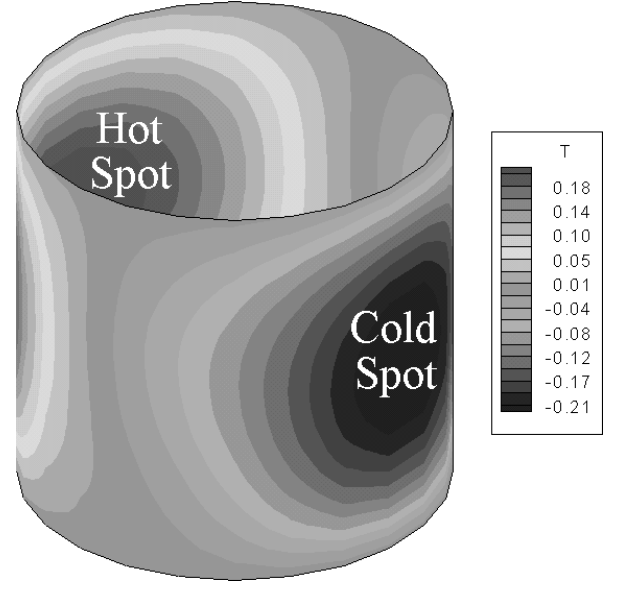

Fig.4c: Temperature disturbances $\mathrm{r}=1 / 2 \mathrm{~A}\left(\mathrm{~A}=1.0, \mathrm{Ma}=110, \mathrm{t}=\mathrm{t}^{*}\right)$

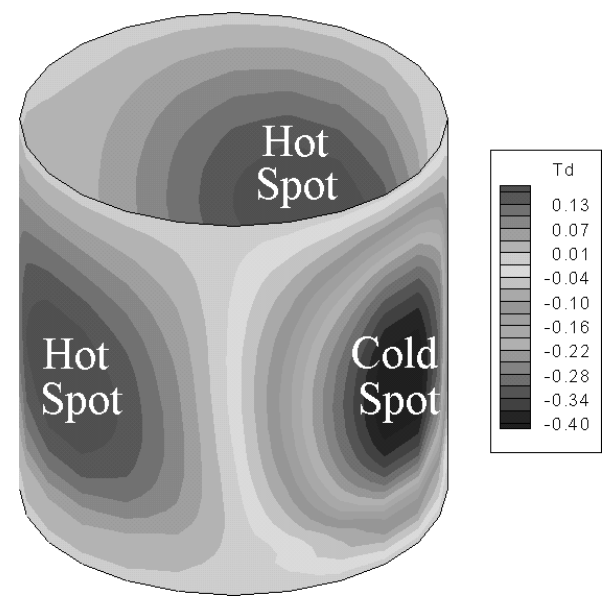

Fig.5c: Temperature disturbances $\mathrm{r}=1 / 2 \mathrm{~A}\left(\mathrm{~A}_{\mathrm{i}}=0.885, \mathrm{Ma}_{\mathrm{i}}=100, \mathrm{t}-\mathrm{t}^{*}=2.6\right)$

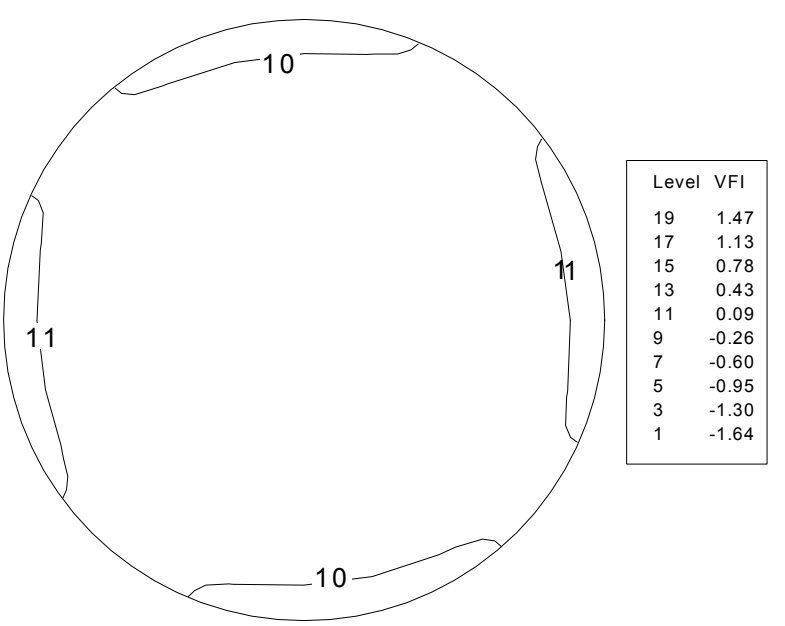

Fig. 9b: azimuthal velocity distribution $\mathrm{z}=0.75\left(\mathrm{~A}_{\mathrm{i}}=0.28, \mathrm{Ma}_{\mathrm{i}}=32, \mathrm{t}-\mathrm{t}^{*}=20.6\right)$

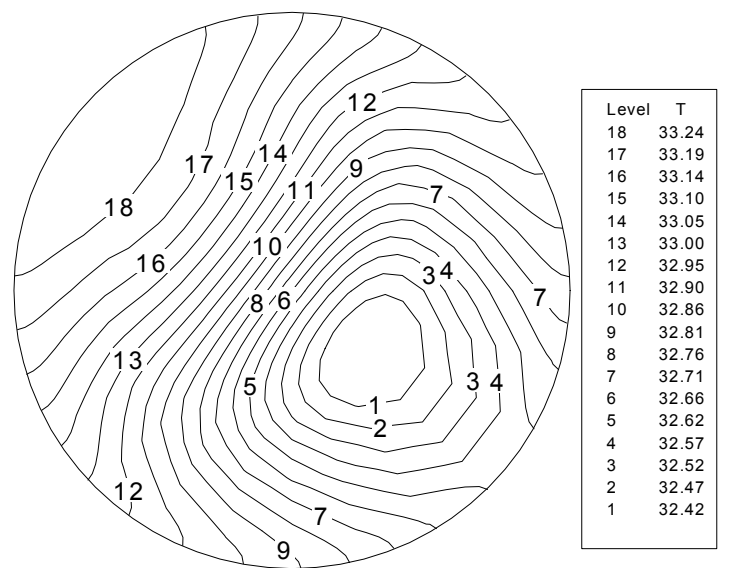

Fig. 4d: Temperature distribution in the section $\mathrm{z}=0.75\left(\mathrm{~A}=1.0, \mathrm{Ma}=110, \mathrm{t}=\mathrm{t}^{*}\right)$

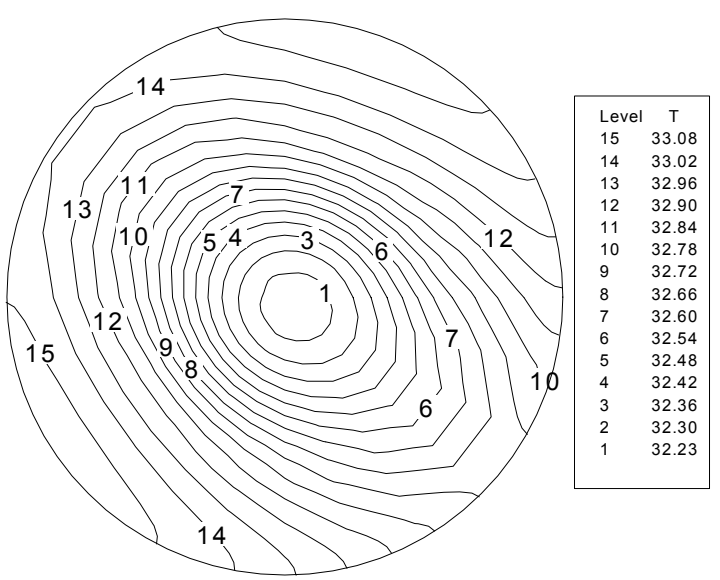

Fig. 5d: Temperature distribution $\mathrm{z}=0.75\left(\mathrm{~A}_{\mathrm{i}}=0.885, \mathrm{Ma}_{\mathrm{i}}=100, \mathrm{t}-\mathrm{t}^{*}=2.6\right)$ 


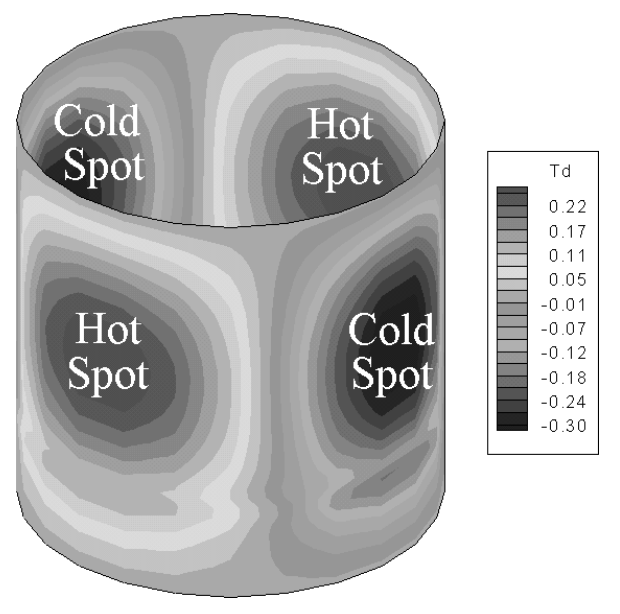

Fig.6c: Temperature disturbances $\mathrm{r}=1 / 2 \mathrm{~A}\left(\mathrm{~A}_{\mathrm{i}}=0.72, \mathrm{Ma}_{\mathrm{i}}=82, \mathrm{t}-\mathrm{t}^{*}=6.2\right)$

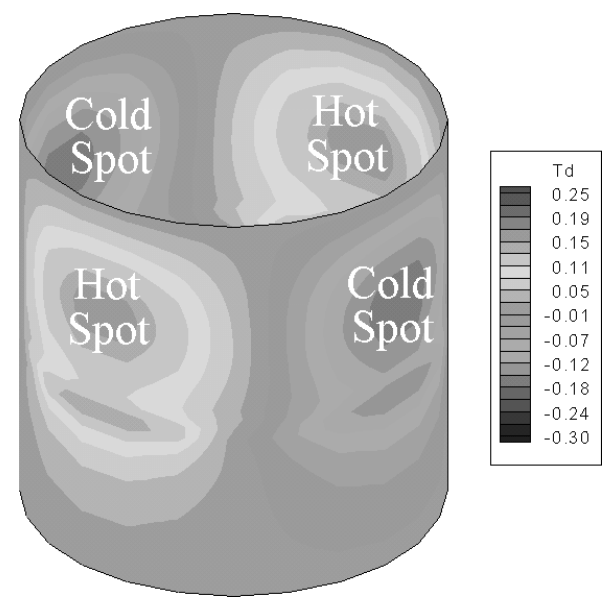

Fig.7c: Temperature disturbances $\mathrm{r}=1 / 2 \mathrm{~A}\left(\mathrm{~A}_{\mathrm{i}}=0.50, \mathrm{Ma}_{\mathrm{i}}=57, \mathrm{t}-\mathrm{t}^{*}=11.9\right)$

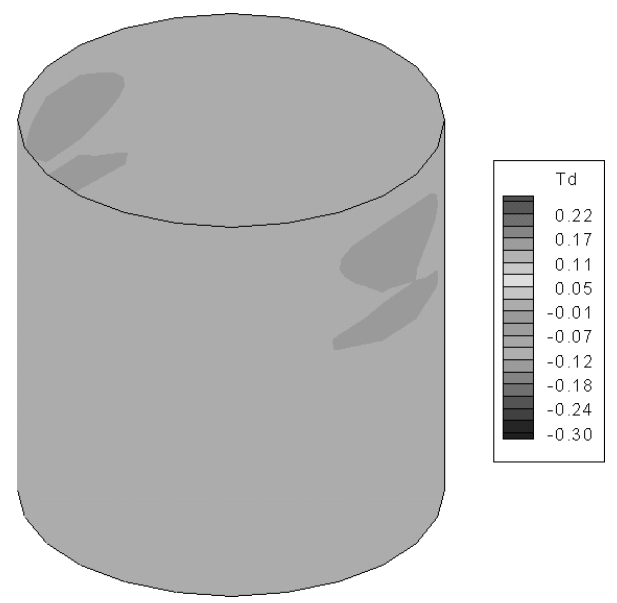

Fig.8c: Temperature disturbances $\mathrm{r}=1 / 2 \mathrm{~A}\left(\mathrm{~A}_{\mathrm{i}}=0.33, \mathrm{Ma}_{\mathrm{i}}=37, \mathrm{t}-\mathrm{t} *=17.5\right)$

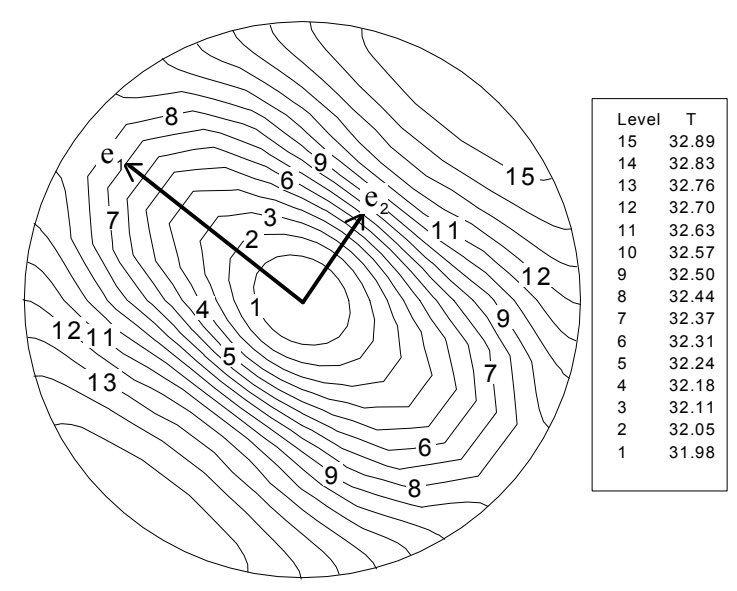

Fig. 6d: Temperature distribution $\mathrm{z}=0.75\left(\mathrm{~A}_{\mathrm{i}}=0.72, \mathrm{Ma}_{\mathrm{i}}=82, \mathrm{t}-\mathrm{t}^{*}=6.2\right)$

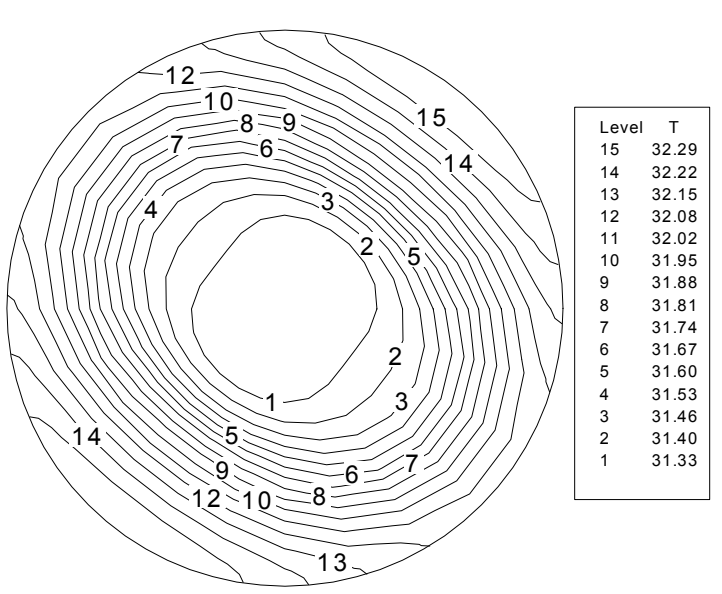

Fig. 7d: Temperature distribution $\mathrm{z}=0.75\left(\mathrm{~A}_{\mathrm{i}}=0.50, \mathrm{Ma}_{\mathrm{i}}=57, \mathrm{t}-\mathrm{t}^{*}=11.9\right)$

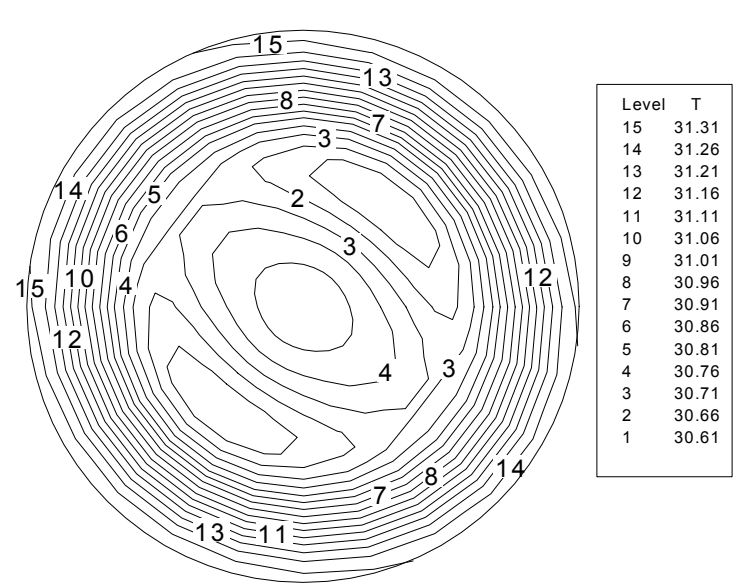

Fig. 8d: Temperature distribution $\mathrm{z}=0.75\left(\mathrm{~A}_{\mathrm{i}}=0.33, \mathrm{Ma}_{\mathrm{i}}=37, \mathrm{t}-\mathrm{t} *=17.5\right)$ 


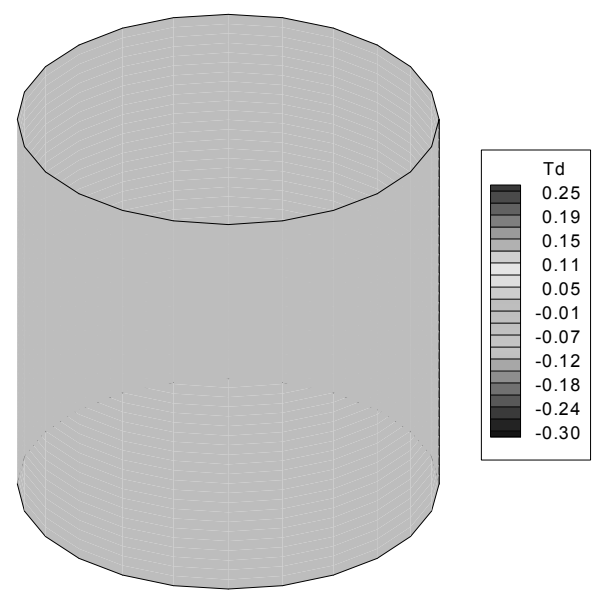

Fig.9c: Temperature disturbances $\mathrm{r}=1 / 2 \mathrm{~A}\left(\mathrm{~A}_{\mathrm{i}}=0.28, \mathrm{Ma}_{\mathrm{i}}=32, \mathrm{t}-\mathrm{t}^{*}=20.6\right)$

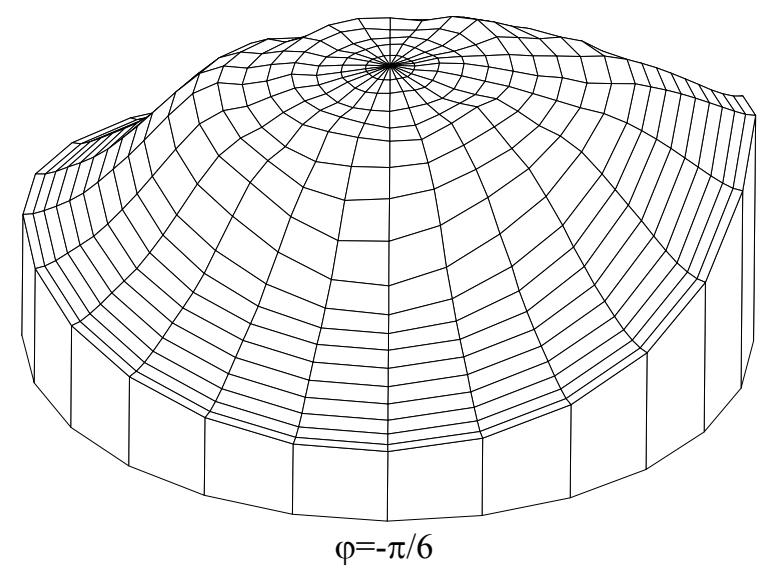

Fig.10a: Crystal/melt interface $\left(\mathrm{A}_{\mathrm{i}}=0.885, \mathrm{Ma}_{\mathrm{i}}=100, \mathrm{t}-\mathrm{t}^{*}=2.6\right)$

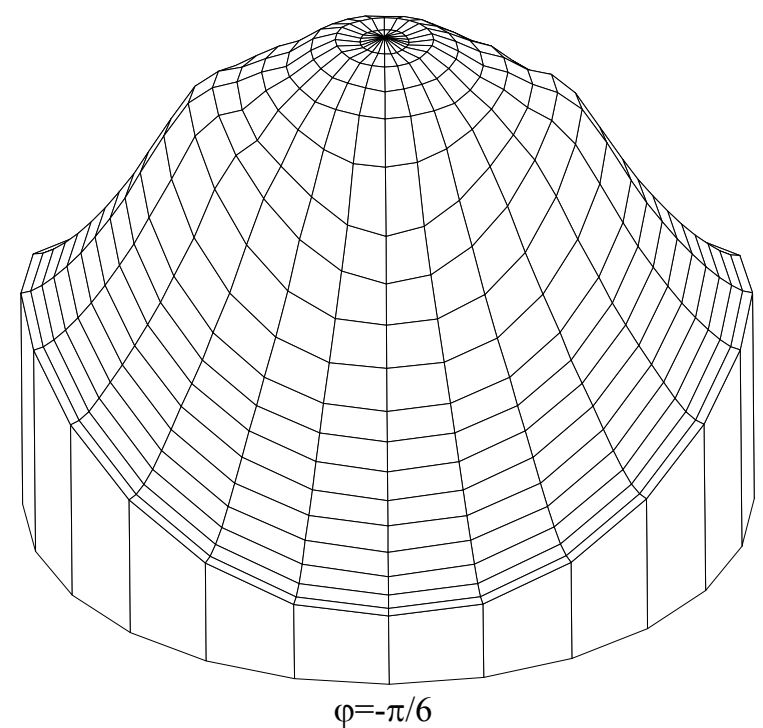

Fig.10c: Crystal $/$ melt interface $\left(\mathrm{A}_{\mathrm{i}}=0.50, \mathrm{Ma}_{\mathrm{i}}=57, \mathrm{t}-\mathrm{t}^{*}=11.9\right)$

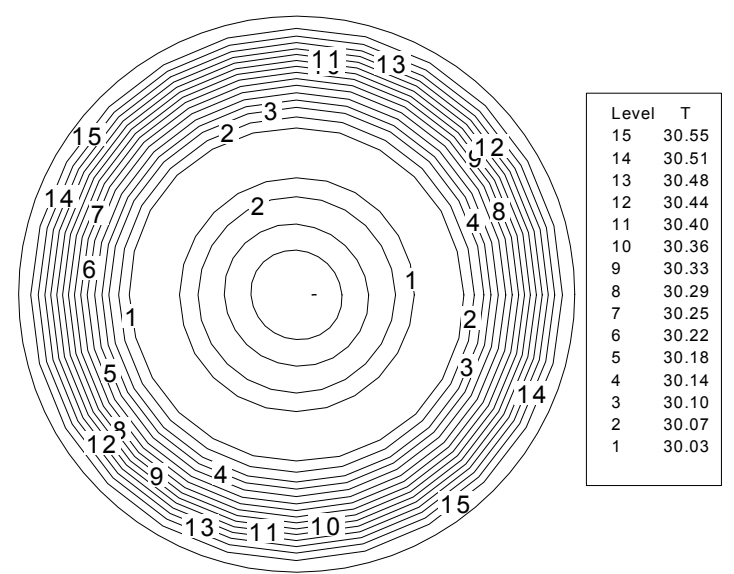

Fig. 9d: Temperature distribution $\mathrm{z}=0.75\left(\mathrm{~A}_{\mathrm{i}}=0.28, \mathrm{Ma}_{\mathrm{i}}=32, \mathrm{t}-\mathrm{t} *=20.6\right)$

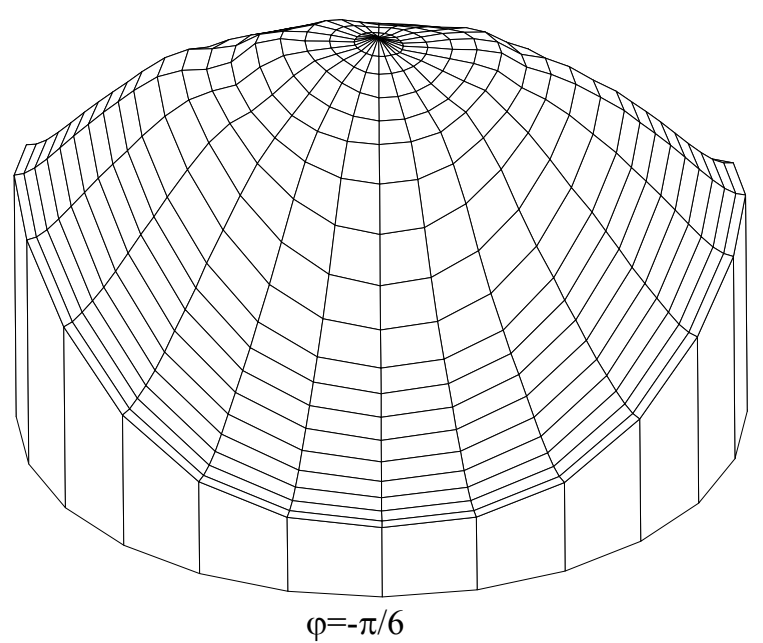

Fig.10b: Crystal $/$ melt interface $\left(\mathrm{A}_{\mathrm{i}}=0.72, \mathrm{Ma}_{\mathrm{i}}=82, \mathrm{t}-\mathrm{t}^{*}=6.2\right)$

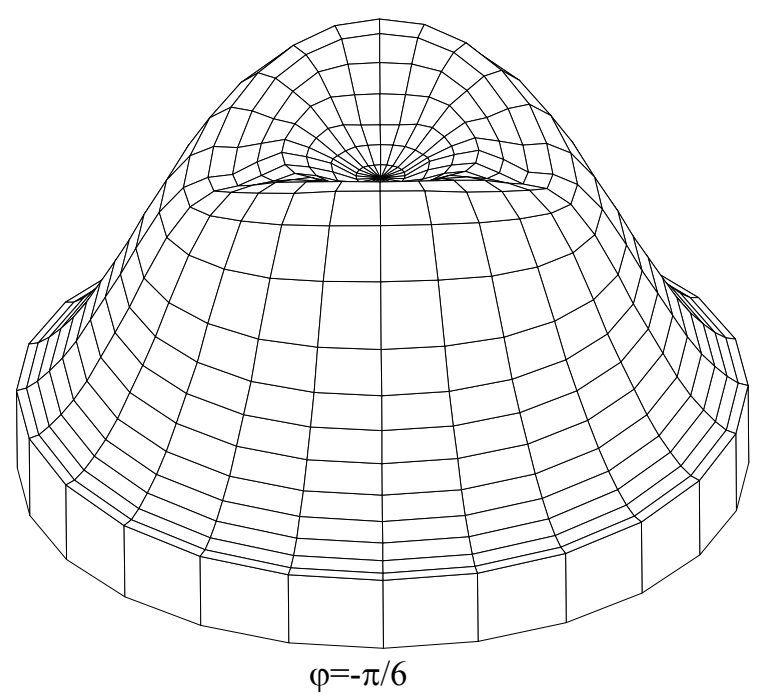

Fig.10d: Crystal/melt interface $\left(\mathrm{A}_{\mathrm{i}}=0.33, \mathrm{Ma}_{\mathrm{i}}=37, \mathrm{t}-\mathrm{t}^{*}=17.5\right)$ 


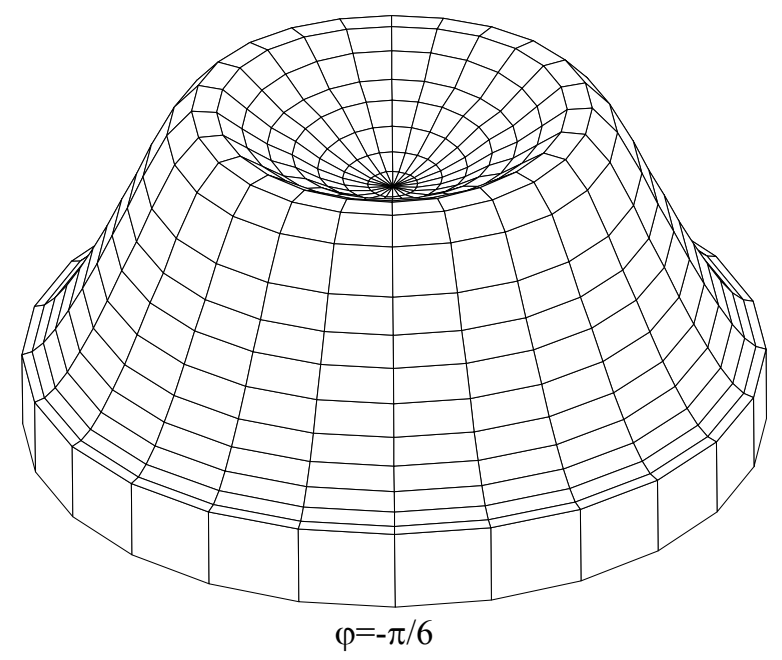

Fig.10e: Crystal/melt interface $\left(\mathrm{A}_{\mathrm{i}}=0.28, \mathrm{Ma}_{\mathrm{i}}=32, \mathrm{t}-\mathrm{t}^{*}=20.6\right)$

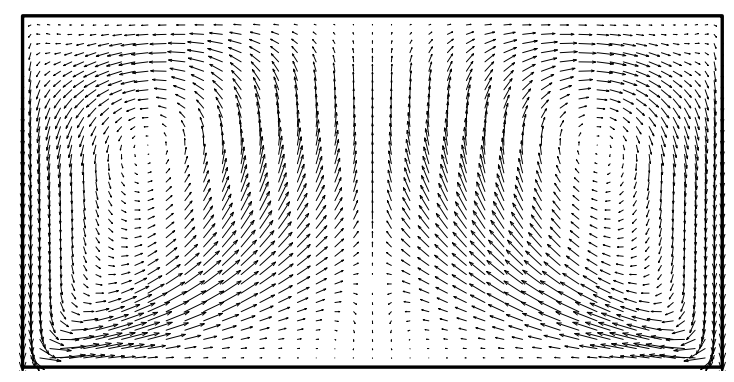

Fig.11a: Flow field in the meridian plane $\varphi=3 \pi / 7\left(\mathrm{~A}=0.50, \mathrm{Ma}=110, \mathrm{t}=\mathrm{t}^{*}\right)$

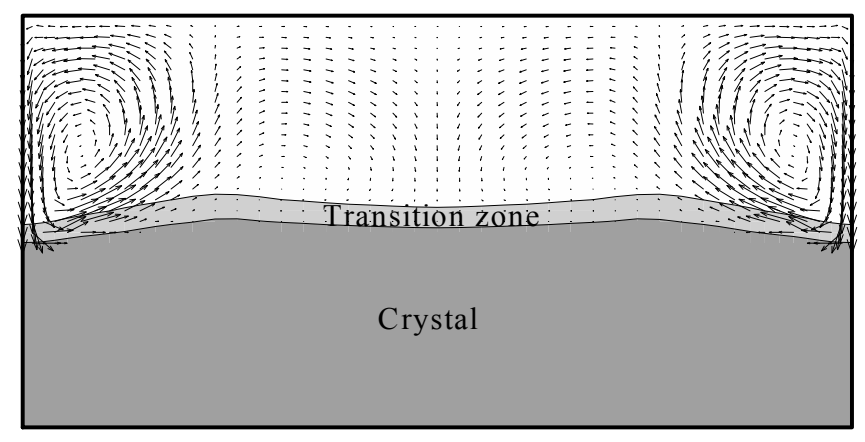

Fig.12a: Flow field in the meridian plane $\varphi=3 \pi / 7\left(\mathrm{~A}_{\mathrm{i}}=0.24, \mathrm{Ma}_{\mathrm{i}}=55, \mathrm{t}-\mathrm{t}^{*}=23\right)$

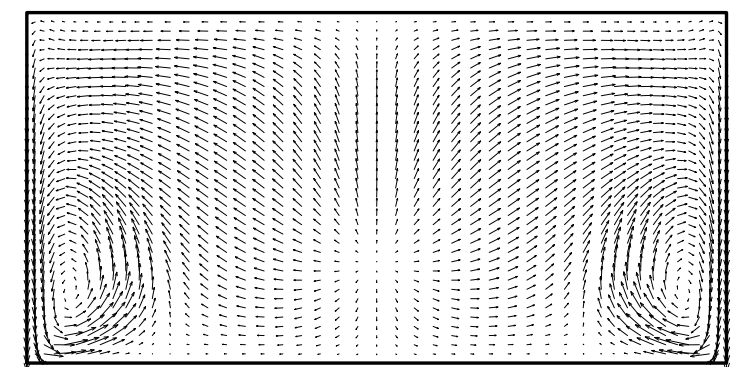

Fig.11b: Flow field in the meridian plane $\varphi=10 \pi / 7\left(\mathrm{~A}=0.50, \mathrm{Ma}=110, \mathrm{t}=\mathrm{t}^{*}\right)$

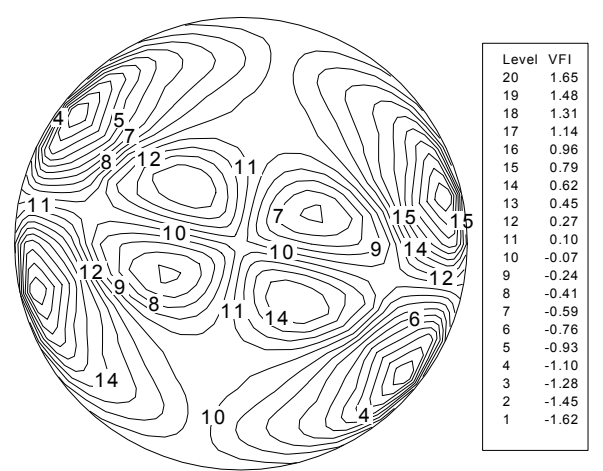

Fig. 12b: azimuthal velocity distribution $\mathrm{z}=0.75\left(\mathrm{~A}_{\mathrm{i}}=0.24, \mathrm{Ma}_{\mathrm{i}}=55, \mathrm{t}-\mathrm{t} *=23\right)$ 


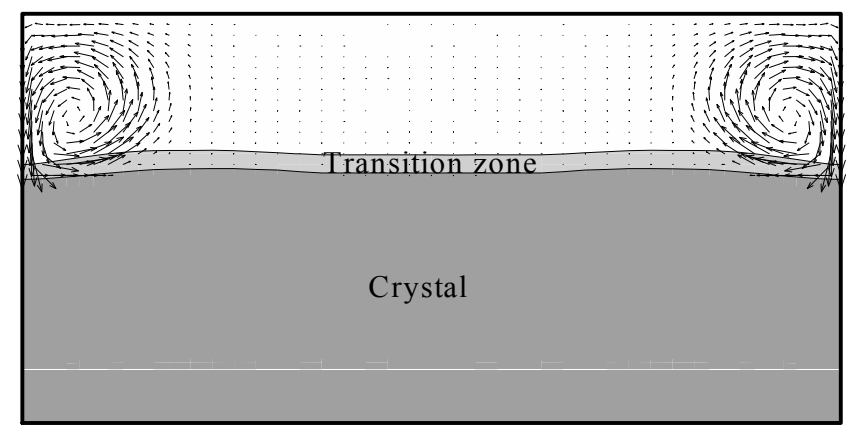

Fig.13a: Flow field in the meridian plane $\varphi=3 \pi / 7 \quad\left(\mathrm{~A}_{\mathrm{i}}=0.18, \mathrm{Ma}_{\mathrm{i}}=41, \mathrm{t}-\mathrm{t}^{*}=27.7\right)$

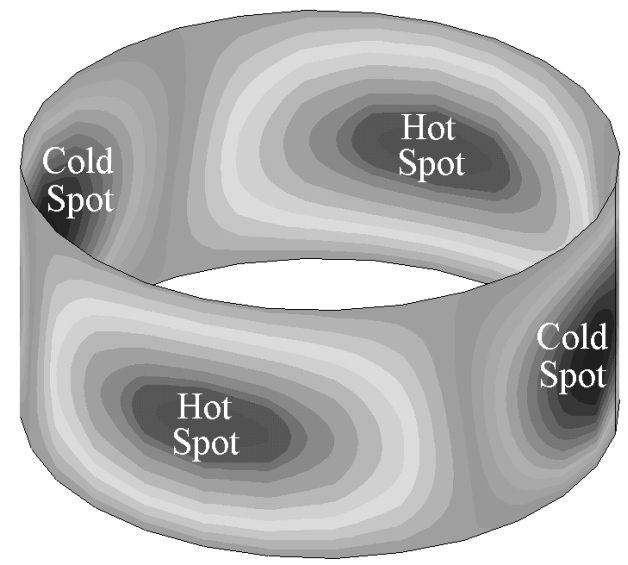

Fig.11c: Temperature disturbances $\mathrm{r}=1 / 2 \mathrm{~A}\left(\mathrm{~A}=0.50, \mathrm{Ma}=110, \mathrm{t}=\mathrm{t}^{*}\right)$

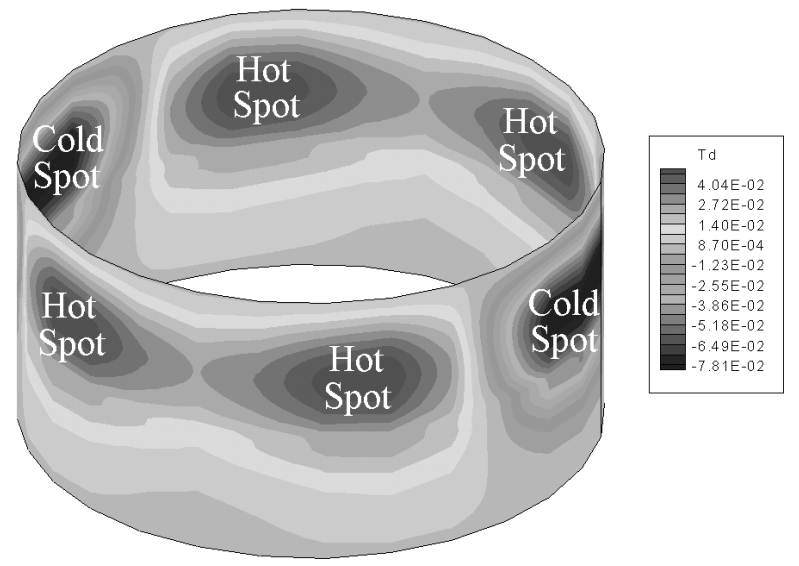

Fig.12c: Temperature disturbances $\mathrm{r}=1 / 2 \mathrm{~A}\left(\mathrm{~A}_{\mathrm{i}}=0.24, \mathrm{Ma}_{\mathrm{i}}=55, \mathrm{t}-\mathrm{t}^{*}=23\right)$

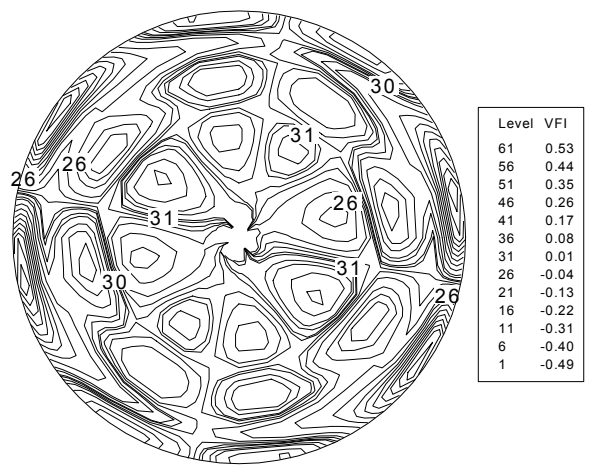

Fig. 13b: azimuthal velocity distribution $\mathrm{z}=0.75\left(\mathrm{~A}_{\mathrm{i}}=0.18, \mathrm{Ma}_{\mathrm{i}}=41, \mathrm{t}-\mathrm{t}^{*}=27.7\right)$

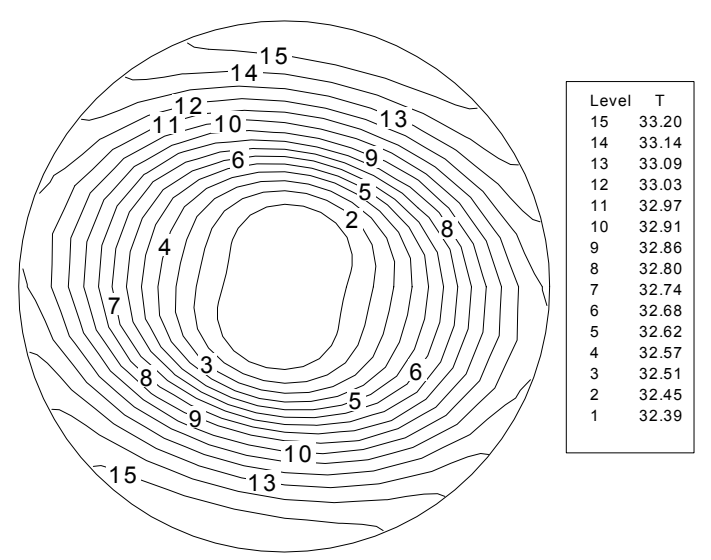

Fig. 11d: Temperature distribution $\mathrm{z}=0.75\left(\mathrm{~A}=0.50, \mathrm{Ma}=110, \mathrm{t}=\mathrm{t}^{*}\right)$

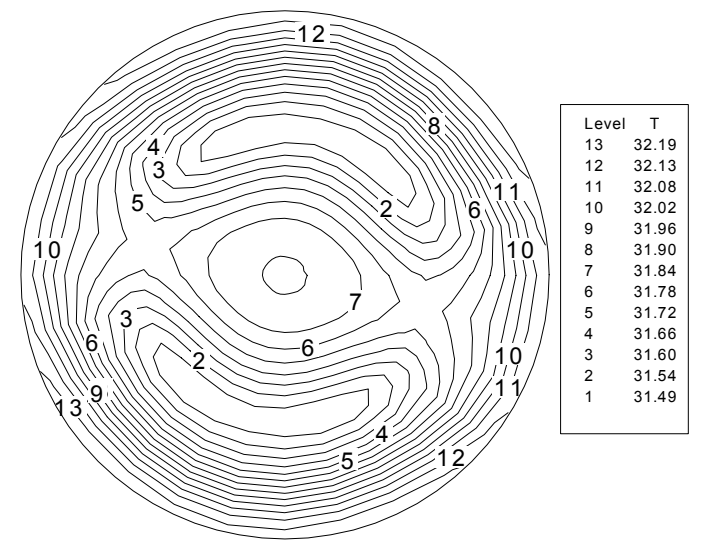

Fig. 12d: Temperature distribution $\mathrm{z}=0.75\left(\mathrm{~A}_{\mathrm{i}}=0.24, \mathrm{Ma}_{\mathrm{i}}=55, \mathrm{t}-\mathrm{t}^{*}=23\right)$ 


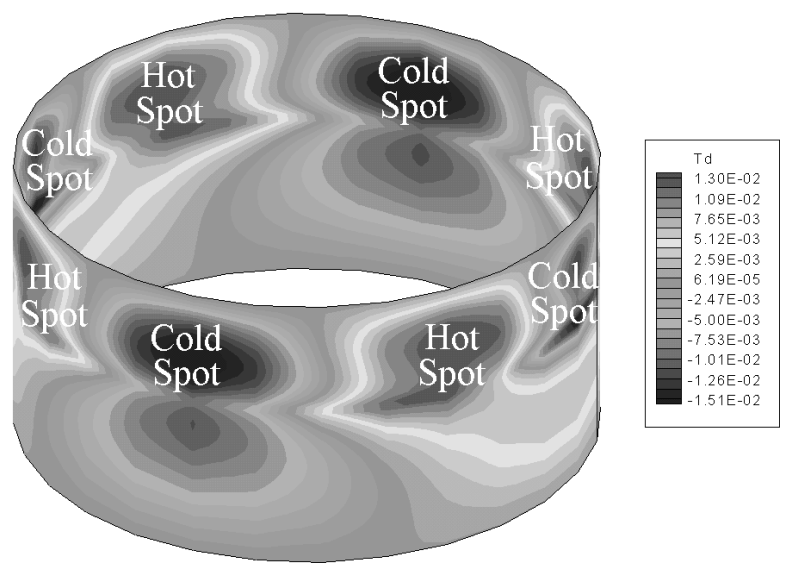

Fig.13c: Temperature disturbances $\mathrm{r}=1 / 2 \mathrm{~A}\left(\mathrm{~A}_{\mathrm{i}}=0.18, \mathrm{Ma}_{\mathrm{i}}=41, \mathrm{t}-\mathrm{t} *=27.7\right)$

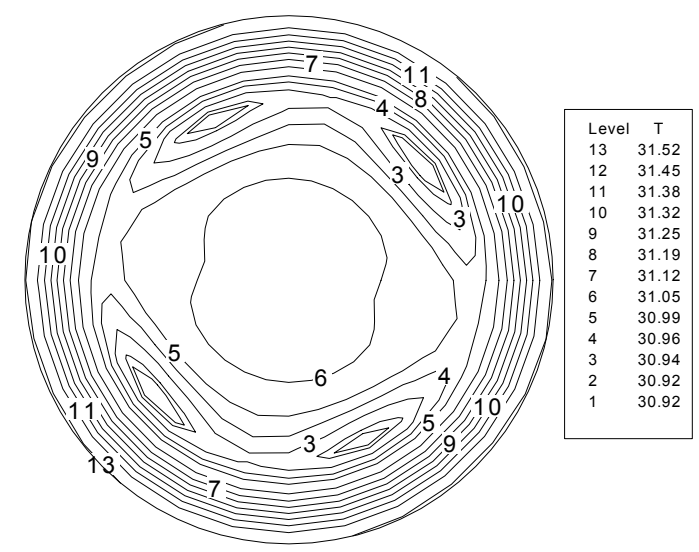

Fig. 13d: Temperature distribution $\mathrm{z}=0.75\left(\mathrm{~A}_{\mathrm{i}}=0.18, \mathrm{Ma}_{\mathrm{i}}=41, \mathrm{t}-\mathrm{t}^{*}=27.7\right)$

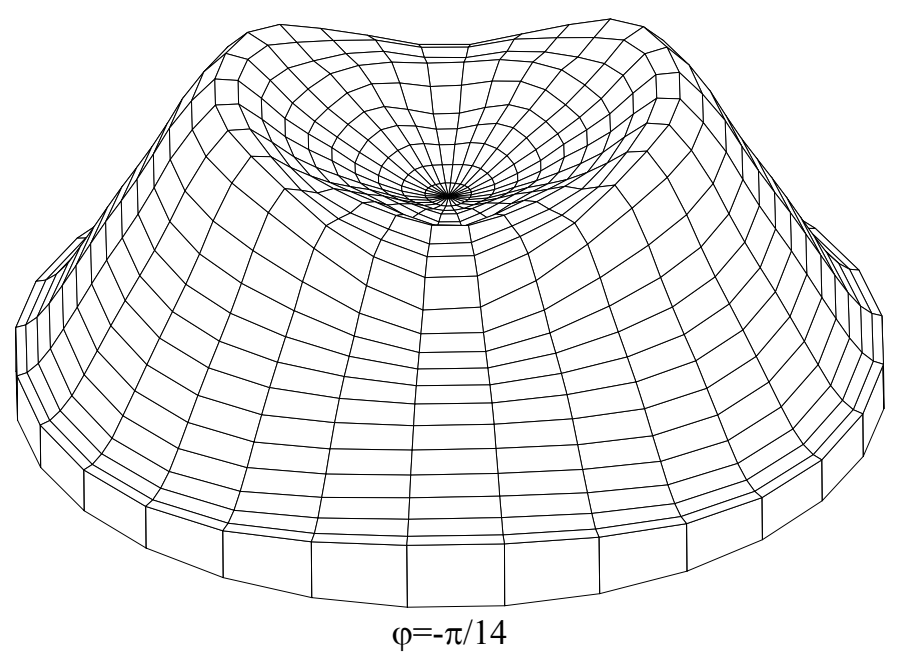

Fig.14a: Crystal/melt interface $\left(\mathrm{A}_{\mathrm{i}}=0.24, \mathrm{Ma}_{\mathrm{i}}=55, \mathrm{t}-\mathrm{t}^{*}=23\right)$

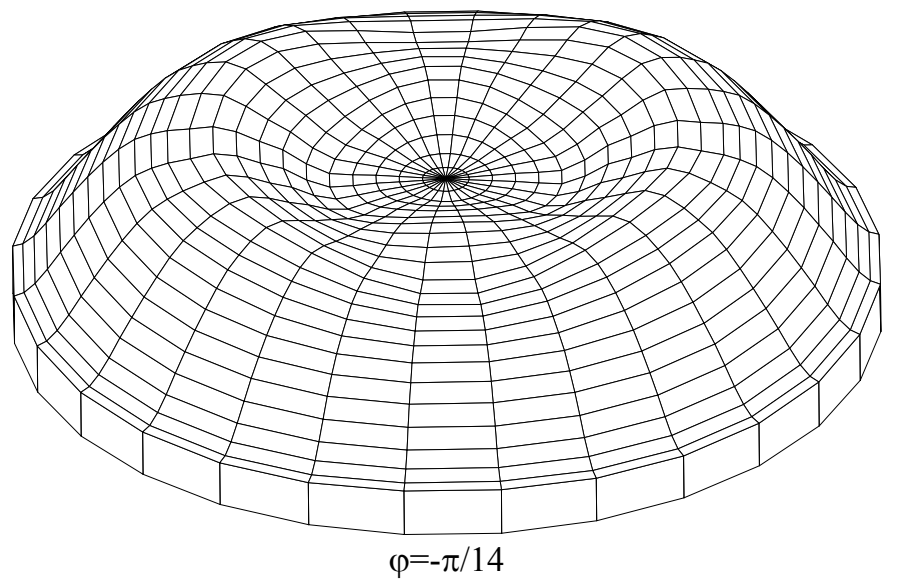

Fig. 14b: Crystal/melt interface $\left(\mathrm{A}_{\mathrm{i}}=0.18, \mathrm{Ma}_{\mathrm{i}}=41, \mathrm{t}-\mathrm{t}^{*}=27.7\right)$ 


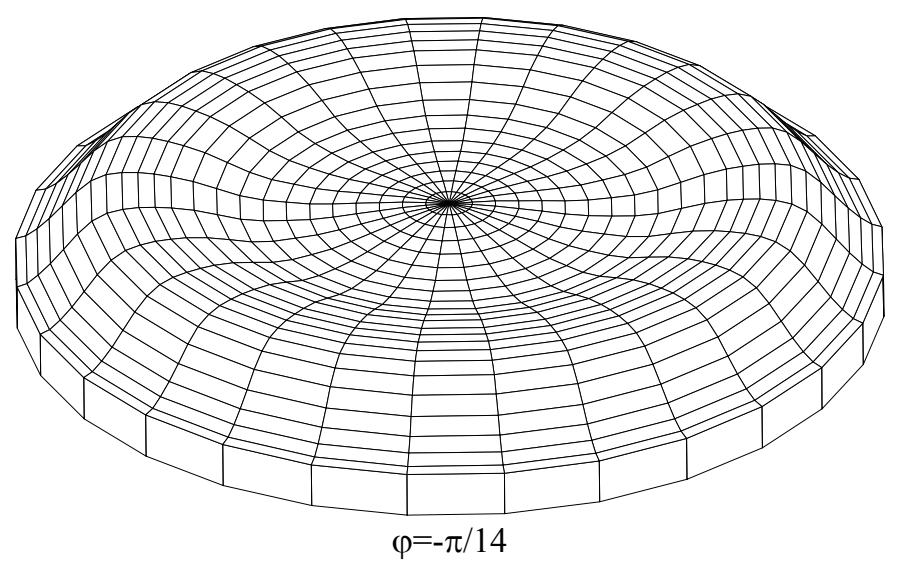

Fig.14c: Crystal/melt interface

$\left(\mathrm{A}_{\mathrm{i}}=0.135, \mathrm{Ma}_{\mathrm{i}}=31, \mathrm{t}-\mathrm{t}^{*}=33.2\right)$ 